\title{
Key driver genes as potential therapeutic targets in renal allograft rejection
}

\author{
Zhengzi Yi, ${ }^{1}$ Karen L. Keung, ${ }^{2,3}$ Li Li, ${ }^{4,5}$ Min Hu, ${ }^{2,6}$ Bo Lu, ${ }^{2}$ Leigh Nicholson, ${ }^{2}$ Elvira Jimenez-Vera, ${ }^{2}$ \\ Madhav C. Menon, ${ }^{1}$ Chengguo Wei, ${ }^{1}$ Stephen Alexander, ${ }^{6,7}$ Barbara Murphy, ${ }^{1}$ Philip J. O'Connell, ${ }^{2,6,8}$ \\ and Weijia Zhang' \\ 'Division of Nephrology, Department of Medicine, Icahn School of Medicine at Mount Sinai, New York, New York, USA. \\ ${ }^{2}$ Centre for Transplant and Renal Research, Westmead Institute for Medical Research, University of Sydney, Sydney, \\ Australia. ${ }^{3}$ Department of Nephrology, Prince of Wales Hospital, Sydney, Australia. ${ }^{4}$ Department of Cenetics and \\ Cenomic Sciences, Icahn School of Medicine at Mount Sinai, New York, New York, USA. ${ }^{5}$ Sema4, Stamford, Connecticut, \\ Connecticut, USA. ${ }^{6}$ Faculty of Medicine and Health, University of Sydney, Sydney, Australia. ${ }^{7}$ Nephrology Department, The \\ Children's Hospital at Westmead, Sydney, Australia. ${ }^{8}$ Department of Nephrology, Westmead Hospital, Sydney, Australia.
}

Authorship note: ZY and KLK are cofirst authors and contributed equally.

\section{Conflict of interest: BM reports} stock in RenalytixAI. WZ reports personal fees from RenalytixAl. $B M, R J O$, and WZ report the US Provisional Patent Application F\&R reference 27527-0134P01, serial number 61/951,651, filed March 2014, "Method for identifying kidney allograft recipients at risk for chronic injury." BM and WZ report the patents US Provisional Patent Application PCT/US2015/038147, "Methods for diagnosing risk of renal allograft fibrosis and rejection"; US Provisional Patent Application US15/321,885, "Method for diagnosing subclinical and clinical acute rejection by analysis of predictive gene sets"; and US Provisional Patent Application PCT/ US2016/065286, "Pretransplant prediction of post-transplant acute rejection." RJO is a consultant for CSL Behring and Vitaeris. RJO has received licensing fees from RenalytixAl for work unrelated to this study. He has a family member who has stock in Medtronic as an employee benefit.

Copyright: (c) 2020, American Society for Clinical Investigation.

Submitted: January 15, 2020

Accepted: June 24, 2020

Published: July 7, 2020.

Reference information: JCI Insight. 2020;5(15):e136220. https://doi.org/10.1172/jci. insight.136220.
Acute rejection (AR) in renal transplantation is an established risk factor for reduced allograft survival. Molecules with regulatory control among immune pathways of AR that are inadequately suppressed, despite standard-of-care immunosuppression, could serve as important targets for therapeutic manipulation to prevent rejection. Here, an integrative, network-based computational strategy incorporating gene expression and genotype data of human renal allograft biopsy tissue was applied, to identify the master regulators - the key driver genes (KDCs) - within dysregulated AR pathways. A 982-meta-gene signature with differential expression in AR versus non-AR was identified from a meta-analysis of microarray data from 735 human kidney allograft biopsy samples across 7 data sets. Fourteen KDGs were derived from this signature. Interrogation of 2 publicly available databases identified compounds with predicted efficacy against individual KDGs or a key driver-based gene set, respectively, which could be repurposed for AR prevention. Minocycline, a tetracycline antibiotic, was chosen for experimental validation in a murine cardiac allograft model of AR. Minocycline attenuated the inflammatory profile of AR compared with controls and when coadministered with immunosuppression prolonged graft survival. This study demonstrates that a network-based strategy, using expression and genotype data to predict KDGs, assists target prioritization for therapeutics in renal allograft rejection.

\section{Introduction}

Acute rejection (AR) currently occurs in approximately $10 \%-20 \%$ of renal transplant recipients in the present era of calcineurin inhibitor-based (CNI-based) immunosuppression and remains a barrier to optimal allograft outcomes. AR can be classified by the mechanisms that underpin the immune-mediated injury as either acute T cell-mediated rejection (TCMR) or antibody-mediated rejection (ABMR), which can also be present simultaneously (mixed AR) in the rejecting allograft. While TCMR is characterized by mononuclear cell infiltration, predominantly $\mathrm{T}$ cells and monocytes/macrophages, of the renal interstitium and tubules (1), the diagnosis of $A B M R$ is more complex and was recently refined to encompass histological, serological, and molecular features (2). Patients with TCMR and/or ABMR are at greater risk of inferior functional and histological outcomes, including chronic rejection and subsequent allograft loss (3-5). Previous studies with rigorous molecular analyses of kidney allograft tissue have shown that differentiating transcripts exist between TCMR and ABMR, but many molecular transcripts are in fact common to both $(6,7)$. These "universal" transcripts were strongly associated with the presence of rejection of any type, and rankings of the top 100 universal rejection transcripts were high in both TCMR-selective and ABMR-selective transcript lists (7). This suggests the processes of rejection are not mutually exclusive and share common nonspecific mechanisms of injury.

Transcriptomic studies in solid organ transplantation have focused on identifying differentially expressed genes (DEGs) in acute allograft rejection compared with healthy controls (8). Heterogeneity of large-scale gene expression profiling studies has been demonstrated across the published literature, allowing meta-analysis of transcriptomic data to increase the statistical power and accuracy 
of expression level estimates $(9,10)$. However, the standard approach of identifying DEGs provides information on the expression change of each gene in isolation and does not help determine the relevance or contribution of each gene to the pathophysiology of the disease under study. The construction of gene coexpression networks has become a useful approach to organize these genes into groups or modules, based on correlation of their transcript expression patterns (11). This is based on the assumption that highly correlated genes are more likely to be part of similar functions and share transcriptional regulation (12). By further incorporating genotyping data, such as with expression quantitative trait loci (eQTLs) mapping, which associates single nucleotide polymorphism (SNP) data with transcript expression, the causal direction between genes can be inferred and predict the master regulators within the gene modules $(13,14)$. These are the key driver genes (KDGs), whose expression directs changes across the network of molecules involved in disease pathways under their regulatory control. Using this approach, predictions of KDGs in Alzheimer's disease, coronary artery disease, and inflammatory bowel disease have been described (15-17). KDGs provide insights into the mechanisms of disease but may also serve as targets for therapeutic manipulation. Here we describe the identification of KDGs in AR in the solid organ transplant setting.

There is an unmet medical need for novel therapeutics in AR, but despite this, clinical trials evaluating candidates for AR prophylaxis are few (18). Beyond the extensive time and financial costs of de novo drug design and development and the low success rates of transitioning a drug from phase I trials to clinical use, the transplant population represents a relatively small therapeutic market. Drug repositioning or repurposing refers to identifying new therapeutic applications for drugs already in clinical use and provides an efficient alternative approach to drug discovery (19). The advent of large-scale gene expression profiling has enabled the identification of molecular changes caused by various drugs and compounds not previously considered and now readily accessible through a number of databases (20). Computational approaches to drug discovery for repurposing include searching bioinformatic and chemoinformatic resources (DrugBank, ChEMBL) to find drugs that alter the expression of individual gene transcripts $(21,22)$, while more advanced strategies facilitate drug predictions against a gene set (Connectivity Map [CMAP], LINCS) $(23,24)$. This latter approach is particularly relevant in complex conditions, such as AR, where the phenotype is a consequence of the altered expression of numerous molecules across various pathways, where suppressing a network of genes rather than a gene in isolation would have greater therapeutic impact. Our group has previously shown the feasibility of using CMAP to predict therapeutic candidates for repositioning in renal allograft fibrosis, based on a meta-analysis-discovered 85-gene signature with experimental validation of 2 of the identified compounds (25), although KDG derivation was not performed.

In this study, we hypothesized that there are molecular pathways in AR that are inadequately suppressed by standard immunosuppression. We describe the identification of KDGs among these alternative activation pathways in AR that may serve as potential targets for AR prevention.

\section{Results}

A differential gene signature of AR demonstrated substantial enrichment of innate immune cell transcripts among the overexpressed genes. The overall experimental design of our study is presented in Figure 1, and a more detailed workflow of the meta-analysis and gene regulatory network construction is shown in Supplemental Figure 1; supplemental material available online with this article; https://doi.org/10.1172/jci. insight.136220DS1.

First, we obtained microarray gene expression data of renal allograft biopsy tissue from the public domain and our own group, totaling 735 samples across 7 data sets (Supplemental Table 1). To discover a meta-signature associated with AR, samples were dichotomized within each data set into AR and non-AR as designated within their original studies (26-31). AR included phenotypes of TCMR (including borderline), ABMR, and mixed (both TCMR and ABMR) cases, from both indication biopsies (which are prompted by a change in the patient's clinical or laboratory parameters) and protocol biopsies (which are taken at predefined intervals following transplantation, independent of kidney function) (Supplemental Table 1). A meta-analysis was performed by combining effect size and $P$ value-based methods as described in Methods and Supplemental Methods, yielding an overlap differentially expressed meta-gene signature of 982 genes ( 565 up- and 417 downregulated DEGs) associated with AR at a false discovery rate (FDR) cutoff of less than 0.05 (Figure 2A, Supplemental Table 2). The Gene Ontology (GO) enrichment analysis 


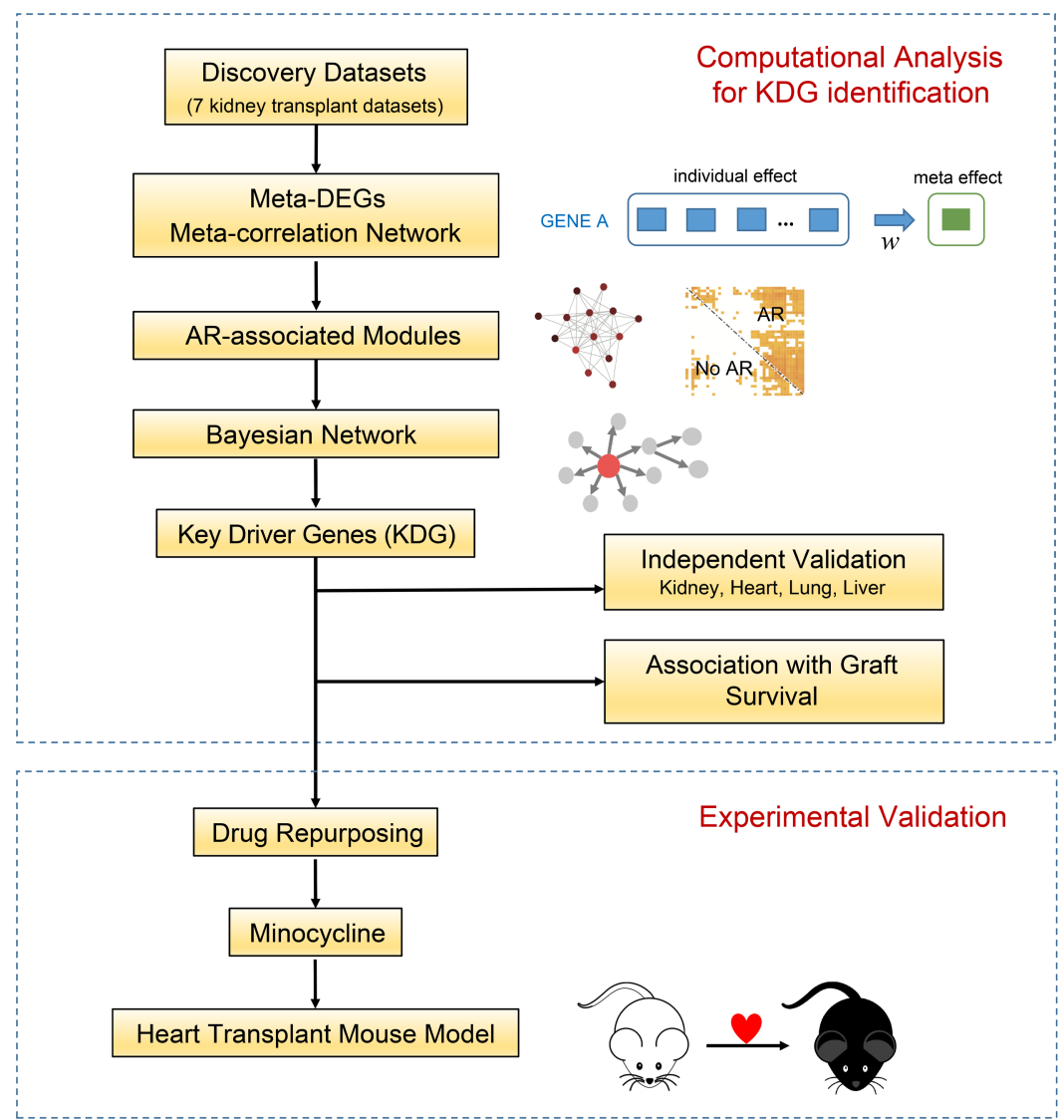

Figure 1. Summary workflow of the computational analyses and experimental validation. Seven publicly available kidney transplant biopsy microarray data sets were a discovery set to identify AR-associated differentially expressed meta-genes. These meta-genes were used to construct meta-coexpression network and AR-associated gene modules. By using the Bayesian network method, KDGs within each of the differential AR modules were identified; their association with AR was validated in additional independent kidney transplant biopsy public data sets. Using the KDGs, the prediction of candidate drugs for repurposing into the AR setting was performed using 2 computational approaches, with only 1 therapeutic agent, minocycline, identified with both strategies. Finally, a murine cardiac allograft model of $A R$ was used to evaluate the effect of minocycline on the inflammatory profile of AR and allograft survival.

of the DEGs by Fisher exact test indicated that the most enriched biological processes represented by the upregulated genes included the immune and defense response, cell activation, and chemotaxis (Figure 2B). Of the downregulated genes, metabolic transport processes were most enriched (Figure 2C).

To investigate which immune cell subsets were represented by the upregulated DEGs, we processed the microarray data sets of mouse immune cell subsets (http://www.immgen.org) and identified genes that were highly expressed by each cell subtype. Using these data, enrichment analysis of upregulated DEGs revealed that cells classically associated with the innate immune system were most strongly represented (Supplemental Figure 2). Specifically, dendritic cells and macrophages and their precursors, monocytes, featured most prominently, followed by $\mathrm{CD} 4^{+} \mathrm{T}$ cells, natural killer $\mathrm{T}$ cells, and natural killer cells (Figure 2D). B cells were not significantly associated with AR.

Fourteen key drivers were identified from the significantly differential gene modules of $A R$. Next, to build the gene expression correlation network for the meta-AR signature, the meta-correlation coefficients (metar) of the expression data of the 982 DEGs in AR samples in 7 data sets were calculated as described in Supplemental Methods, and only those correlations with $\mid$ meta- $r \mid>0.6$ and FDR-adjusted $P<0.05$ were included for network analysis. The Markov Cluster Algorithm (MCL) for clustering based on correlation coefficients was performed on the meta-correlation matrix of DEGs; the top 10 modules were derived and annotated with the most enriched GO functions in Figure 3A. We observed that the up- and downregulated meta-genes segregated into different modules.

To identify the correlation modules specifically associated with AR samples but not the non-AR samples, we compared the overall difference of gene connectivity of each module between AR and non-AR 
A

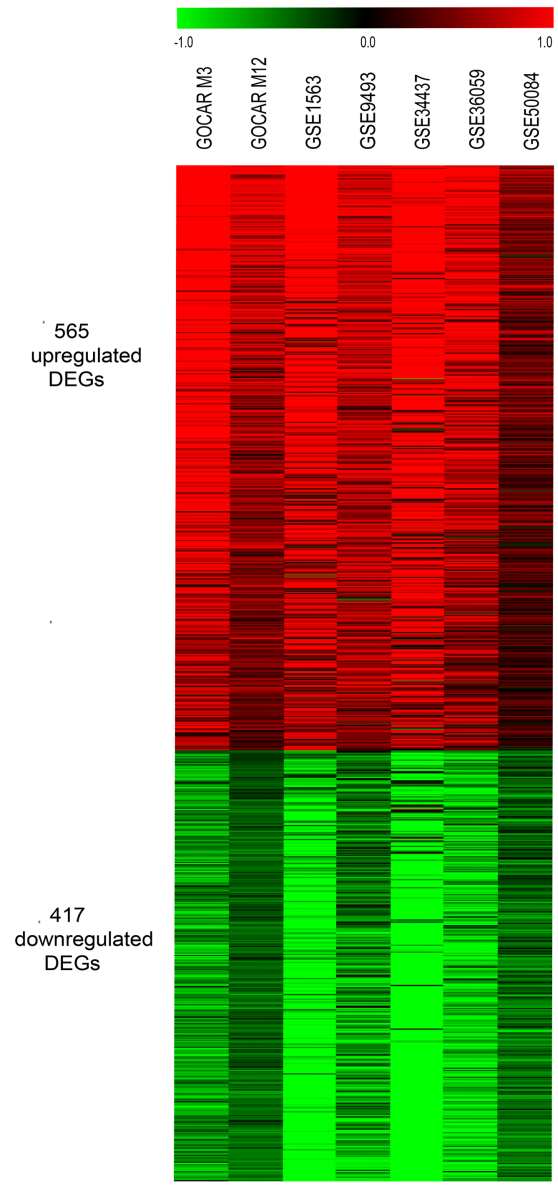

B

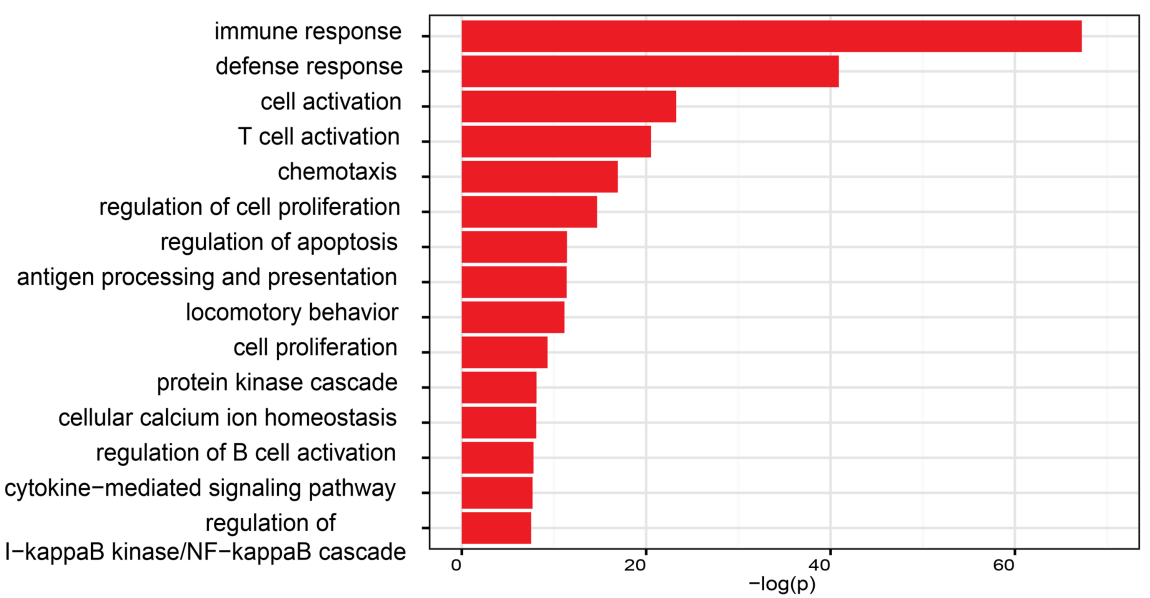

C

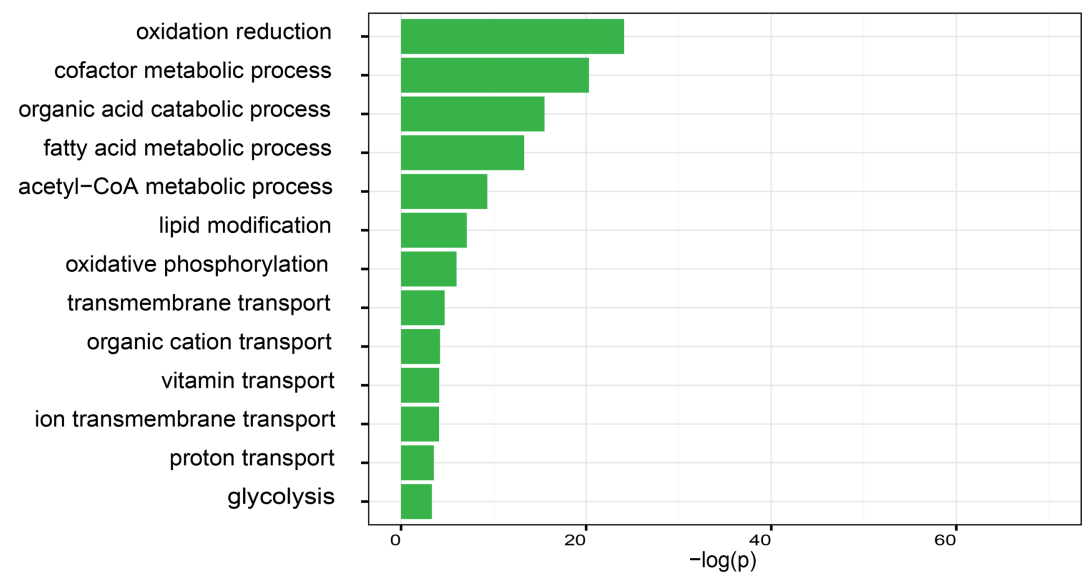

D

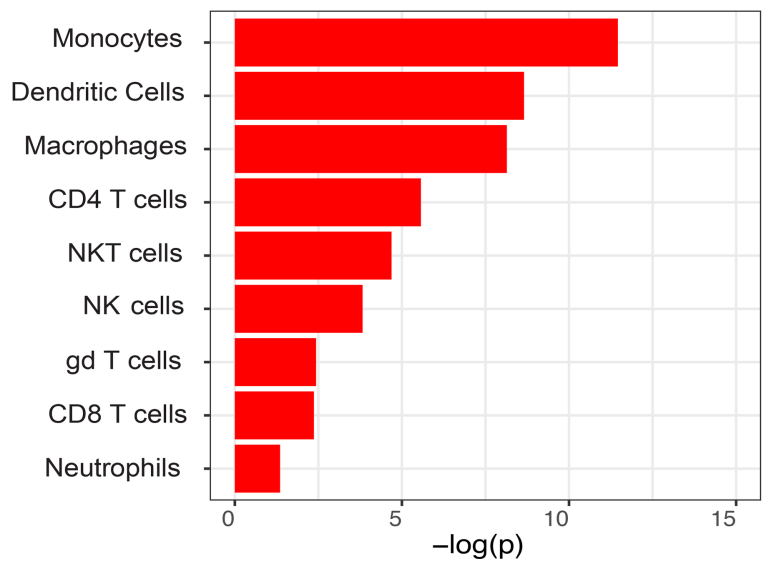

Figure 2. Heatmap representation of the $\mathbf{9 8 2}$ differentially expressed metagenes in AR and functional enrichment analysis. (A) Heatmap demonstrating expression level of the 982 DEGs in each of the 7 public data sets included in the meta-analysis. The heatmap values are represented as effect size (Hedges' g) as depicted in the color scale bar above the heatmap. Green is indicative of downregulation (reduced expression) and red indicative of upregulation (increased expression) of each gene in AR compared with non-AR samples within each data set. (B and C) GO enrichment analysis identified that the most enriched biological processes represented by the upregulated genes (B) include immune response, cell activation, and chemotaxis. The downregulated genes were enriched in metabolism and transport processes (C). Negative log of the $P$ value, $-\log (P)$, is presented, with higher $-\log (P)$ values indicating greater statistical significance. (D) Innate immune cell populations were identified among the upregulated DEGs by cell enrichment analysis based on ImmGen database. Bar chart of significantly enriched cell types is ordered by significance, $-\log (P)$ values.

samples and identified differential modules at a $P$ value cutoff of less than 0.05 by 1000 random permutations test (Figure 3B and Supplemental Figure 3A). Interestingly, all differential modules (modules 1, 3 , $4,7,8,10$ ) were those composed of upregulated DEGs, with modules 1,3 , and 4 consisting of the largest number of genes, and the immune response was identified as the most enriched biological process in each of these modules. Because the assumption of gene coexpression network analysis is that genes clustered by similarity in expression levels are likely to participate in similar functions, differences in dominant biological processes represented by each module were expected (Figure 3A). Consistent with this, cell activation was strongly enriched in module 1 , inflammation and chemotaxis in module 3 , and antigen presentation in module 4, although none of these processes was exclusive to any single module. Modules 2, 5, 6, and 9 , 
comprising downregulated meta-genes, showed no differential connectivity for AR compared with non-AR samples (Supplemental Figure 3A).

Incorporating eQTL data from our Genomics of Chronic Allograft Dysfunction (GoCAR) study, the directional Bayesian network of gene coregulation was built for each differential module to infer the causal relationship between genes. Using a key driver gene algorithm previously described (15) based on the number of out-degrees and downstream genes (Supplemental Figure 3B), 14 KDGs were derived from 5 of the 6 significantly differential modules of AR (Table 1). Of the $14 \mathrm{KDGs}, 12$ belonged to the largest 3 modules, 6 of which belonged to module 1, including caspase-1 (CASP1), which yielded the highest number of both downstream counts and out-degrees of all the KDGs (Supplemental Table 3). CASP1 was also identified as the global driver of module 1 because it was not downstream to any other genes within this module (Supplemental Figure 3B). The KDGs and their closely connected module members are depicted in Figure 4.

Overexpression of KDGs in $A R$ was supported in additional renal and heart transplant data sets but not in liver and lung data sets. Validation of the overexpression of the $14 \mathrm{KDGs}$ was sought in additional kidney transplant biopsy data sets in AR versus non-AR samples and also explored in heart, liver, and lung transplant data sets (Supplemental Table 1) (32-42).

Across 5 additional external and independent renal data sets, a high rate of significantly differential expression of the KDGs was observed. This was moderate in the heart, while few of the KDGs were overexpressed in liver and lung data sets (Figure 5, A and B). The discriminatory ability of the KDG gene set to differentiate AR from non-AR samples in these renal data sets was also evaluated using the geometric mean of the expression of KDGs, and a good performance was achieved, with the area under the receiver operating characteristic (ROC) curve (AUC) of AR detection ranging between 0.71 and 0.92 with a median value of 0.8 (Figure 5C). It also performed similarly in the heart allograft data sets (AUC $=0.78-0.91$ ) (Figure 5D) but less accurately in the liver and lung data sets (liver AUC $=0.41-0.51$, lung AUC $=0.5-0.69$ ) (Supplemental Figure 4).

Key driver expression correlates with renal allograft loss. Because AR is a risk factor for reduced renal allograft survival, the correlation of KDG overexpression with graft loss was further assessed in 2 data sets, the first being the GoCAR data set GSE57387 (26) comprising protocol biopsies obtained 3 months posttransplant $(n=159)$, which was included in our meta-analyses, and GSE21374 $(n=282)(32)$, an independent validation data set comprising indication biopsies performed between 4 months and 37 years posttransplant. Within each data set, patient samples were divided equally based on the survival probability from Cox regression model of association of the expression of $14 \mathrm{KDG}$ with graft survival, and graft survival was compared by Kaplan-Meier analysis. KDG expression was significantly associated with allograft attrition in both data sets $(P=1.82 \mathrm{e}-03$ in GSE57387 and $P=2.11 \mathrm{e}-08$ in GSE21374) (Figure 6, A and C). The ROC curve analysis of graft loss prediction using KDG expression across the samples in each data set further demonstrated this gene set was strongly predictive of graft loss in the first and second years posttransplant in GSE57387 (AUC 0.913 and 0.913, respectively) and in the first and second years postbiopsy in GSE21374 (AUC 0.971 and 0.889, respectively) (Figure 6, B and D).

Minocycline is identified by drug-repositioning strategies as a potential candidate to suppress key driver expression. Drugs with U.S. Food and Drug Administration (FDA) approval in other disease settings were sought to identify therapeutics that could suppress key driver expression and therefore be repurposed for prophylaxis of AR, as an adjunct to established immunosuppression. A 42-gene set from the largest modules (modules 1, 3, and 4), composed of 12 KDGs and 30 secondary genes with large out-degrees, was inputted into CMAP (Supplemental Table 4) (23). CMAP is a large-scale perturbation database of transcriptional profiles, derived from the treatment of cultured human cells with a library of 1309 drugs or small bioactive molecules, most of which are FDA approved. For each compound a therapeutic ranking score was generated (see Methods). At a $P$ value of less than $0.05,67$ compounds were discovered with a negative correlation score, suggesting an inverse drug-disease relationship (Supplemental Table 5A). Interrogation of DrugBank (22), a database that provides drug data with drug target information, was also performed to identify FDA-approved drugs that inhibit the individual KDGs (Supplemental Table 5B). Among the results, minocycline was identified as an inhibitor of CASP1, the global driver of the largest differential gene module of AR (Supplemental Table 3) and was the only therapeutic agent discovered in both the DrugBank and CMAP interrogation with the KDGs (Supplemental Figure 5). It is a semisynthetic tetracycline antibiotic, although its primary clinical application at present is for acne treatment. Beyond its antimicrobial effects, minocycline has pleiotropic antiinflammatory and 
A
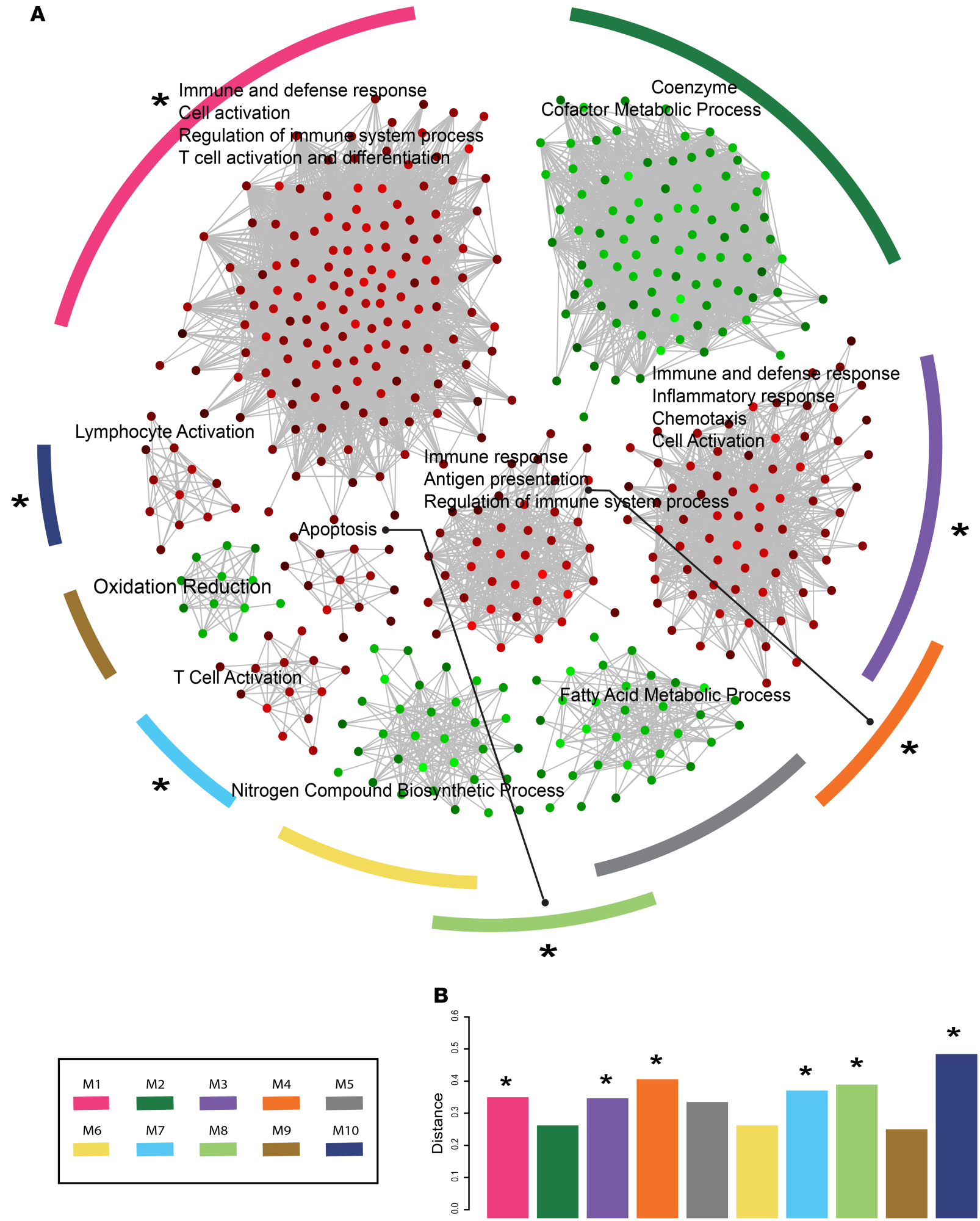

Figure 3. Coexpression modules based on meta-genes and differential modules between AR versus non-AR patients. (A) Top 10 modules (M1-M10) and their primary GO biological processes. Six of the 10 modules were able to differentiate between AR from non-AR patients, as highlighted with an asterisk (permutation test $P<0.05$ ). The largest modules comprising the greatest number of DEGs were 1,3 , and 4 . Each node represents a gene, with the color denoting the degree of upregulation (red) or downregulation (green). (B) Bar plot in lower panel shows connectivity distance between AR patients and nonAR patients. Differential modules are marked with asterisks. 
Table 1. The 14 KDGs from the differential modules of AR

\begin{tabular}{|c|c|c|c|c|}
\hline Gene symbol & RefSeq accession & Full name & Meta-effect size ${ }^{A}$ & Meta- $P$ value \\
\hline CASP1 & NM_033295 & Caspase-1 & 1.09 & $5.41 E-07$ \\
\hline CD3D & NM_001040651 & Delta subunit of the CD3 receptor & 1.05 & $1.78 \mathrm{E}-06$ \\
\hline CRLF3 & NM_015986 & Cytokine-like receptor factor 3 & 0.90 & $2.11 E-04$ \\
\hline EVI2A & NM_001003927 & Ecotropic viral integration site $2 \mathrm{a}$ & 1.18 & $7.68 \mathrm{E}-05$ \\
\hline GPR65 & NM_003608 & G-coupled protein receptor 65 & 1.02 & $9.56 \mathrm{E}-08$ \\
\hline HCK & NM_001172129 & Hematopoietic cell kinase & 1.14 & $2.16 \mathrm{E}-09$ \\
\hline LCP1 & NM_002298 & Lymphocyte cytosolic protein 1 & 1.16 & $3.12 \mathrm{E}-06$ \\
\hline MNDA & NM_002432 & $\begin{array}{c}\text { Myeloid cell nuclear differentiation } \\
\text { antigen }\end{array}$ & 0.96 & 4.74E-07 \\
\hline MY01F & NM_012335 & Unconventional myosin if & 1.16 & $2.12 E-09$ \\
\hline PLEK & NM_002664 & Plekstrin & 0.96 & $3.16 \mathrm{E}-08$ \\
\hline PSMB10 & NM_002801 & Proteasome beta subunit type 10 & 1.01 & 1.47E-07 \\
\hline PSME1 & NM_006263 & $\begin{array}{l}\text { Proteasome activator complex subunit } \\
\qquad 1\end{array}$ & 0.55 & $3.83 \mathrm{E}-03$ \\
\hline RASSF2 & NM_170774 & $\begin{array}{l}\text { Ras association domain-containing } \\
\text { protein } 2\end{array}$ & 1.08 & 2.97E-05 \\
\hline VAV1 & NM_001258207 & $\begin{array}{l}\text { Vav guanine nucleotide exchange } \\
\text { factor } 1\end{array}$ & 0.97 & $2.52 \mathrm{E}-07$ \\
\hline
\end{tabular}

Meta- $P$ values are adjusted by FDR correction method. ${ }^{A}$ Hedges' $g$, which estimates the mean difference between the AR and non-AR group.

immunomodulatory properties demonstrated across various experimental inflammatory disease models, including multiple sclerosis (43), inflammatory bowel disease (44), and diabetic nephropathy (45). In solid organ transplantation, its protective effects have been described in allograft rodent models of allograft ischemia/reperfusion injury (46) but have not yet been examined in AR.

Therefore, minocycline was selected as the repurposed drug candidate for AR and tested in an established murine BALB/c to C57BL6 heterotopic cardiac allograft model of AR representing a full MHC mismatch, to examine its impact on KDG expression and more specifically the inflammatory profile of AR and allograft survival (Figure 7A).

Minocycline reduces expression of Caspase- 1 and limits the inflammatory profile of AR. Beyond its identification in DrugBank as a CASP1 inhibitor, discovery of minocycline in CMAP against the key driver-based gene set suggested that expression of other KDGs may also be reduced by minocycline. The mRNA transcript of the $14 \mathrm{KDG}$ was therefore examined in a murine cardiac allograft model comparing allogeneic allografts from recipient mice treated with twice-daily minocycline alone $(n=9)$ or saline $(n=9)$ administered via the IP route, starting 36 hours before transplantation through to POD4 when allografts were still viable (that is, allograft heartbeat still manually palpable) but rejection was underway, at which time animals were euthanized and allograft tissue was harvested. Treatment was started 36 hours before transplant to establish drug presence preoperatively with consideration to its short half-life in rodents (2-3 hours). This contrasts to that in humans, where it is $12-18$ hours, which is similar to that of established immunosuppressive agents in clinical practice (e.g., tacrolimus and mycophenolate). Syngeneic C57BL6 allografts were used as controls $(n=4)$. In addition to CASP1 $(P=0.028)$, the key drivers HCK $(P=0.041), \operatorname{CD} 3 \mathrm{D}(P=0.049)$, and EVI2A $(P=0.038)$ were significantly decreased (Figure $7 \mathrm{~B})$ in the minocycline treatment group compared with saline treatment, and there was a trend toward decreased expression of a number of other key drivers (Supplemental Figure 6A).

To assess its impact on the inflammatory profile of AR and proinflammatory cytokine and chemokine gene expression, histology and immune cell infiltration were also assessed in these allografts. Minocycline treatment resulted in significantly lower levels of expression of chemokines CCL5 ( $P$ $=0.006)$ and CXCL9 $(P=0.005)$ compared with saline treatment alone (Supplemental Figure 6B), as well as a reduction in IL-2 $(P=0.009)$ and IFN- $\gamma(P=0.003), 2$ cytokines associated with $\mathrm{T}$ cell priming (Figure $7 \mathrm{C})$. IL-1 $\beta$, which requires cleavage by CASP1 to its mature proinflammatory form $(P=0.081)$ (Supplemental Figure $6 \mathrm{~B})$, and the proinflammatory cytokine TNF- $\alpha(P=0.077)$ trended toward but did not reach the level of statistical significance (Figure 7C). 
M1

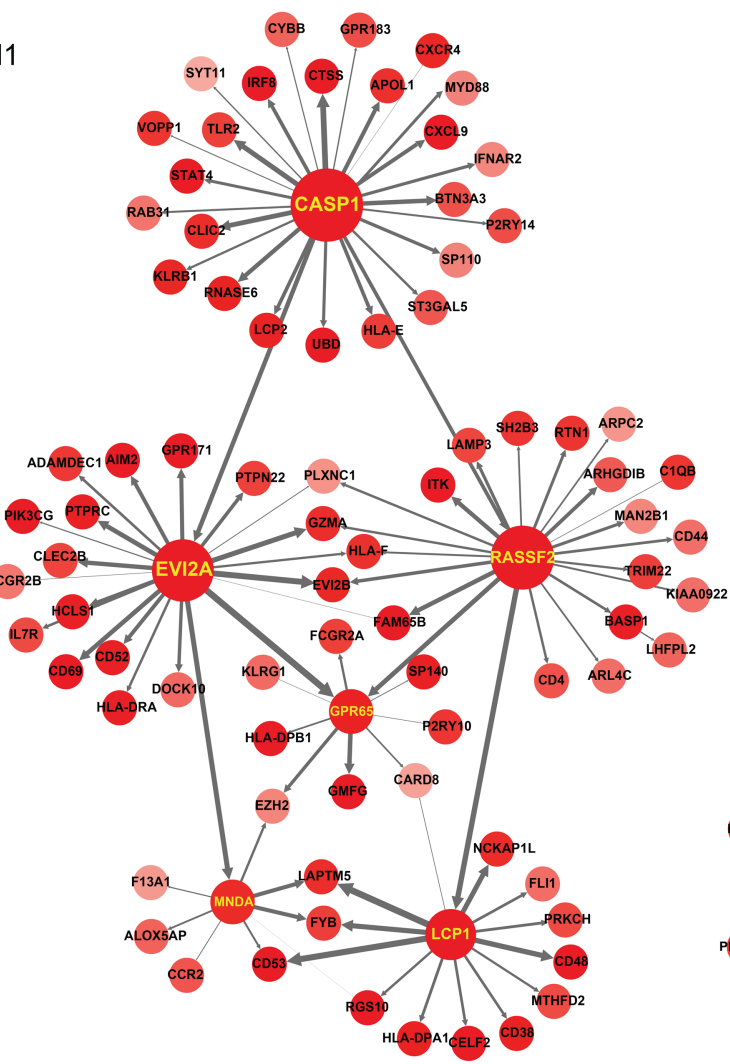

M8

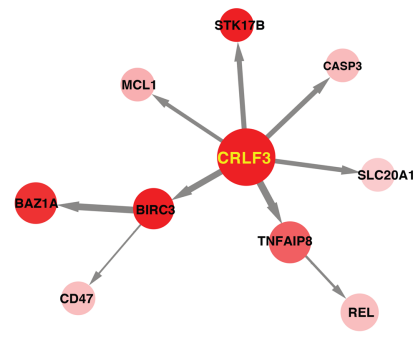

M3

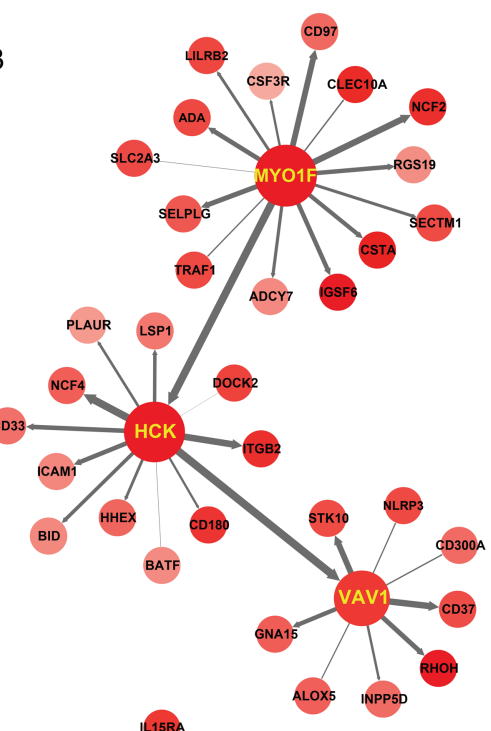

M4

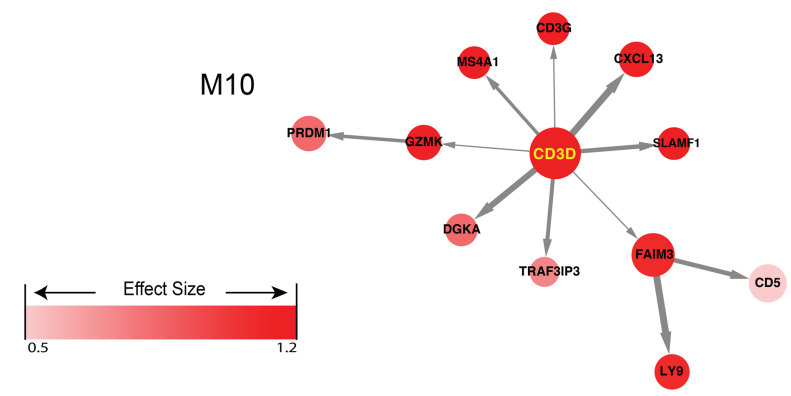

Figure 4. KDGs in AR differential modules. Each colored node represents a meta-gene, and the KDGs are represented with yellow text. The color of each node represents its effect size, reflecting its relative expression level in AR as depicted by the color scale bar at the bottom. The arrows signify the direction of the relationship between 2 genes as predicted by Bayesian network, such that the arrow tip points toward the gene that is downstream of the other. Width of lines indicates strength of correlation between 2 nodes. Due to the size of the modules, only the first layer of downstream genes that were directly connected to key drivers in M1, M3, and M4 are illustrated, although all module members are shown for M8 and M10. In M1, the largest of the modules, since CASP1 was not downstream to any other KDGs in its module, it was identified as the global driver here. Furthermore, CASP1 also had the largest number of out-degrees and downstream gene count of all the KDGs. M7, although regarded as a differential module from connectivity permutation test between AR and non-AR, did not yield a KDC because none of its members satisfied the downstream counts and out-degrees threshold criteria.

Because mRNAs are subject to posttranslational regulatory mechanisms, selective evaluation of IFN- $\gamma$ and TNF- $\alpha$ protein expression in mouse sera from the same experimental animals was conducted to determine whether these changes were observed downstream, using cytometric bead array. Consistent with their respective mRNA expression profiles within the graft, serum protein levels of IFN- $\gamma$ were significantly reduced in minocycline-treated animals compared with the saline-treated group $(P=0.005)$, and TNF- $\alpha$ serum protein levels were consistent with the mRNA expression data, where there was no significant difference between groups $(P=0.139)$ (Figure 7D).

Since this in vivo model is mechanistically driven by cell-mediated rejection, we specifically examined the allografts for $\mathrm{T}$ cells and macrophage infiltration because they comprise the majority of the 
A

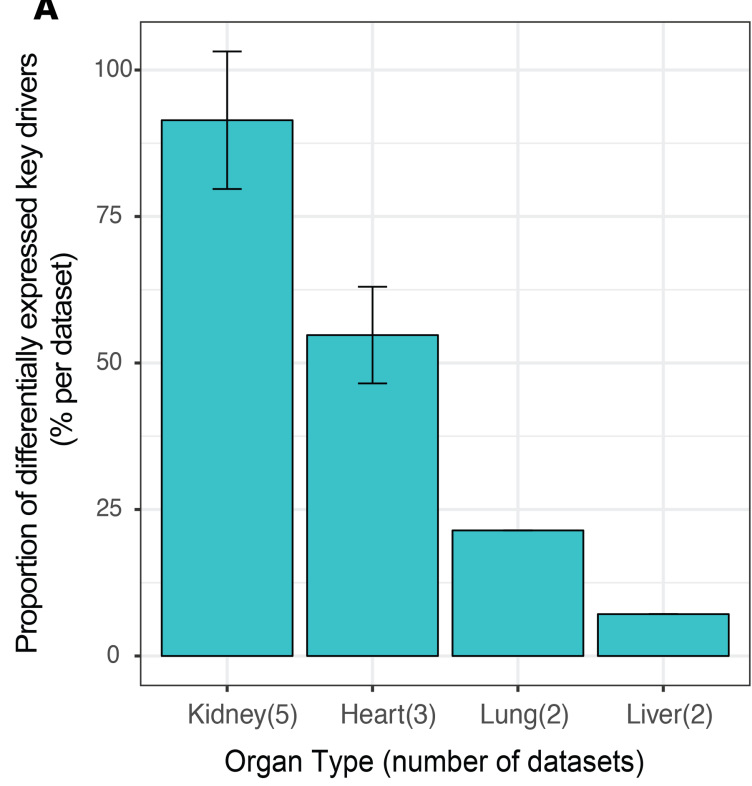

C



B
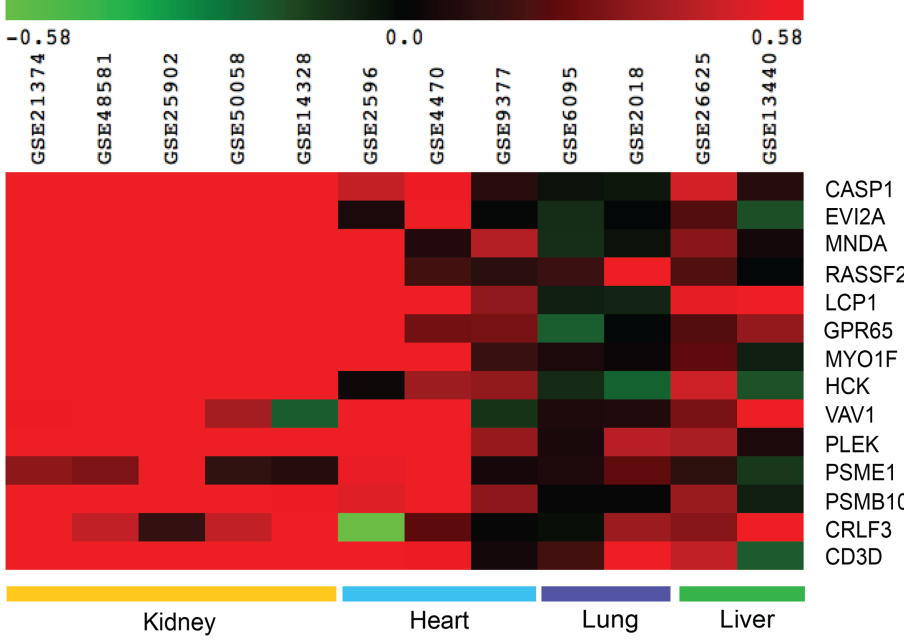

D

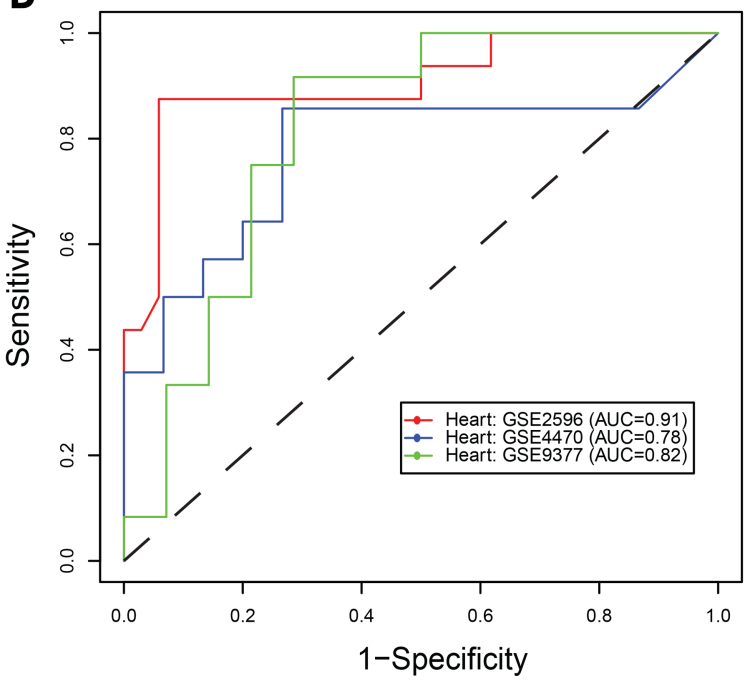

Figure 5. Validation of the overexpression of the 14 KDGs across additional kidney and other solid organ data sets of allograft rejection. (A) Average percentage of significant DEGs in independent public studies from kidney, heart, lung, and liver overlapping with 14 key drivers. Bar graph demonstrating the number of KDGs that were significantly upregulated between AR and non-AR samples across each data set, expressed as an average percentage for each given organ type. A high rate of KDG upregulation was identified in additional kidney data sets and moderate in heart data sets. Few of the KDGs were upregulated in lung and liver allograft data sets. The bar charts represent the percentage of key drivers that are differentially expressed, expressed as a mean per data set for each solid organ type, and the error bars represent standard deviation within each organ type. (B) Heatmap of log (fold change) of 14 KDCs in individual data sets in validation sets. Red color is indicative of higher expression in AR samples, and green color is indicative of lower expression in AR samples. (C and D) Cood discriminatory capacity of the 14-KDG set for AR versus non-AR was observed across the 5 external kidney data sets (C) and heart data sets (D) as demonstrated by the area under the ROC curve (AUC) ranging between 0.71 and 0.92 and 0.78 and 0.91 , respectively, using the geometric mean of expression of the $14 \mathrm{KDCs}$. GSE, gene series expression data set ID from the Gene Expression Omnibus (GEO).

immune cell burden observed in TCMR (47). Consistent with the chemokine and cytokine transcript expression results, the immunohistochemistry staining (Figure 7E) and quantification demonstrated reduced cell infiltrates in the minocycline-treated allografts, with significantly fewer $\mathrm{CD}^{+} \mathrm{T}$ cells $(P=$ $0.001)$ and galectin-3-expressing macrophages $(P=0.0003)$ present compared with those that received saline. Furthermore, the infiltration of both cell types in the minocycline treatment group was not significantly different from that in the syngeneic group (T cells, $P=0.298$; macrophages, $P=0.848$ ) (Figure $7 \mathrm{~F}$ ).

To summarize, minocycline treatment diminished inflammation in the cardiac allografts of mice, with a significant reduction in $\mathrm{T}$ cell and macrophage infiltration and proinflammatory cytokine and chemokine transcript expression. 
A

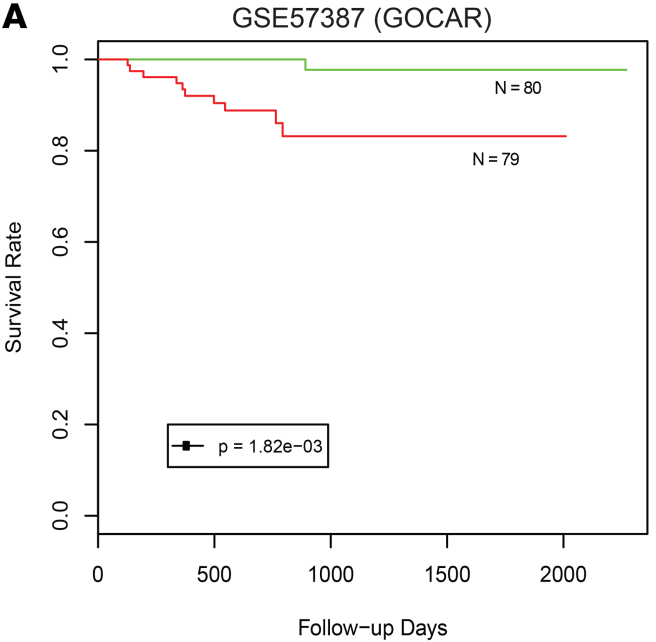

C

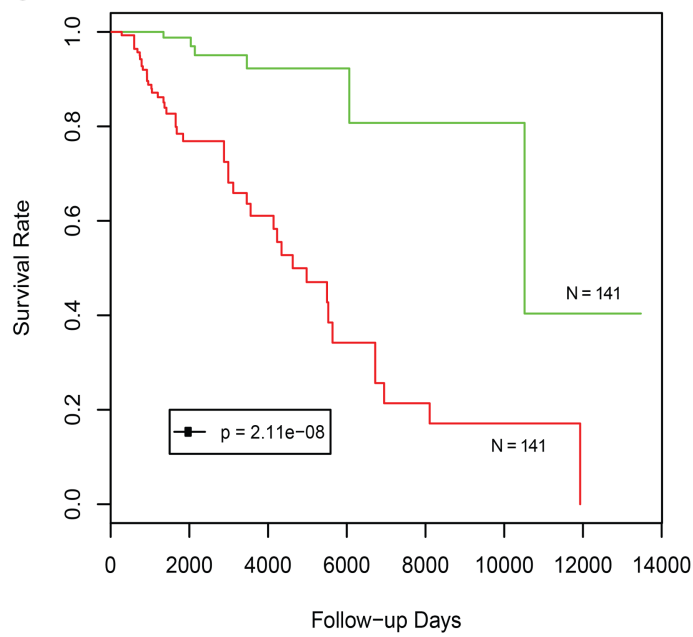

B

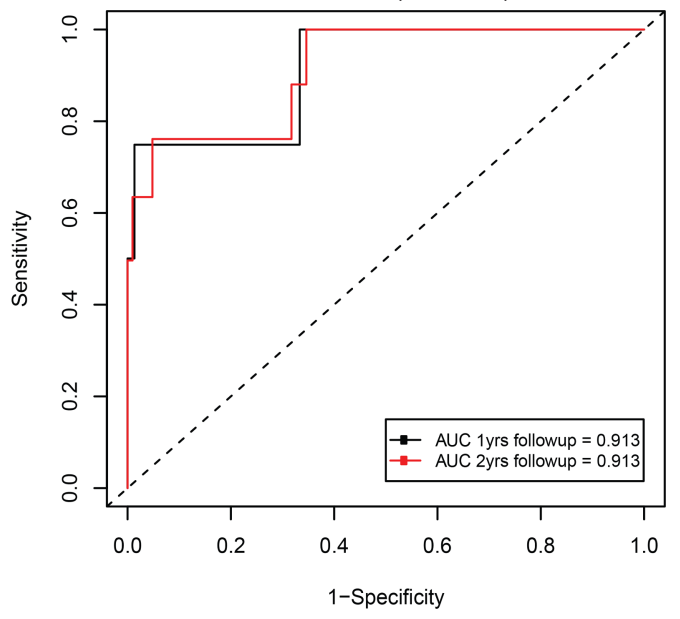

D

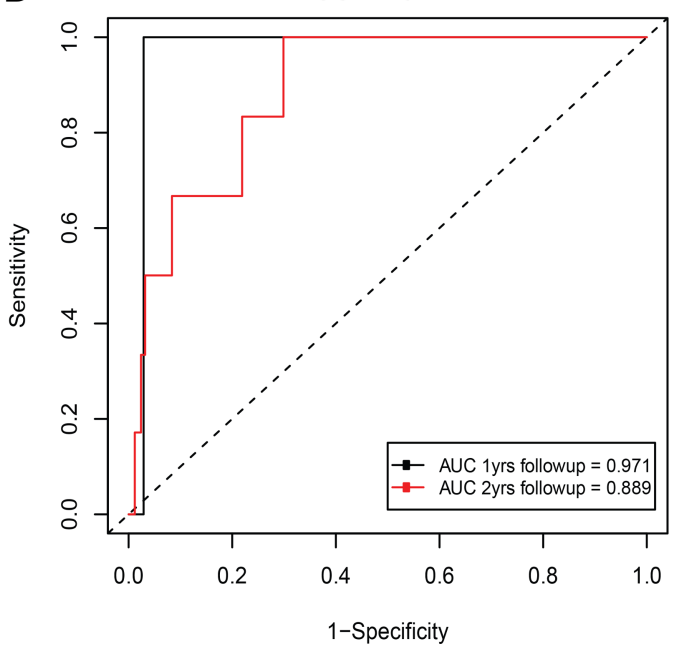

Figure 6. KDG set correlates with renal allograft survival in $\mathbf{2}$ renal allograft transcript data sets. (A) The Kaplan-Meier curve of death-censored graft loss with the kidney transplant recipients in GSE57387 (an internal discovery GoCAR data set composed of protocol biopsies performed 3 months posttransplant) stratified by the median fitted value of 14 key drivers from Cox regression model. $(P=1.82 \mathrm{e}-03)$. (B) The ROC for prediction of graft survival in the GoCAR study at 1 (black curve, AUC $=0.913$ ) and 2 (red curve, AUC $=0.913$ ) years posttransplant. (C) Kaplan-Meier analysis $(P=2.11$ e-08) and $(D)$ ROC plot (1-year postbiopsy follow-up survival: AUC = 0.971, 2-year postbiopsy follow-up survival: $A U C=0.889$ ) using the 14 key drivers in GSE21374 (an external independent data set composed of for-cause biopsies).

Immunosuppression with additional minocycline prolongs allograft survival. Next, the effect of minocycline monotherapy on allograft survival was examined (Supplemental Figure 7A). Kaplan-Meier survival analysis showed minocycline treatment $(n=5)$ compared with saline $(n=5)$ did not prolong allograft survival: minocycline group median survival time (MST) 8 days (range 8-9) versus saline group MST 8 days (range 7-9) $(P=0.614)$ (Supplemental Figure 7B)

Because the KDGs were derived from AR alternative activation pathways in patients receiving standard immunosuppression, we hypothesized that key driver inhibition is indicated as an additive strategy to established immunosuppression to limit rejection. Given our experience with cytotoxic T lymphocyteassociated protein 4-immunoglobulin (CTLA4-Ig) and its current use in clinical kidney transplantation, we used a single IP dose of CTLA4-Ig on POD2 to provide baseline immunosuppression in addition to the minocycline. Its humanized version, belatacept, is FDA approved for AR prophylaxis in renal transplantation (48) as an alternative to CNIs for suppressing T cell activation.

We assessed the effects of CTLA4-Ig with minocycline or with saline treatment. First, we performed histological examination of allografts of animals that received minocycline or saline through to POD14, in combination with single-dose CTLA4-Ig (Figure 8A). All allografts were still viable at 
A

Start minocycline/saline (36 hrs pretransplantation) IP twice daily to POD4

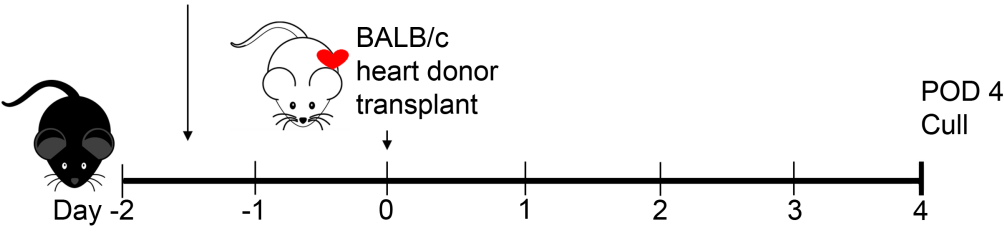

B
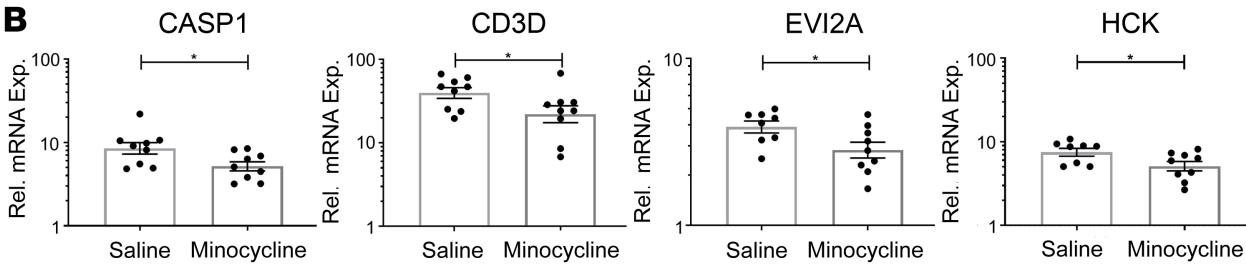

$\mathbf{F}$



Galectin-3

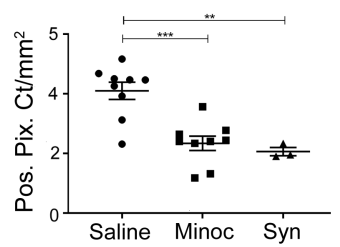

E
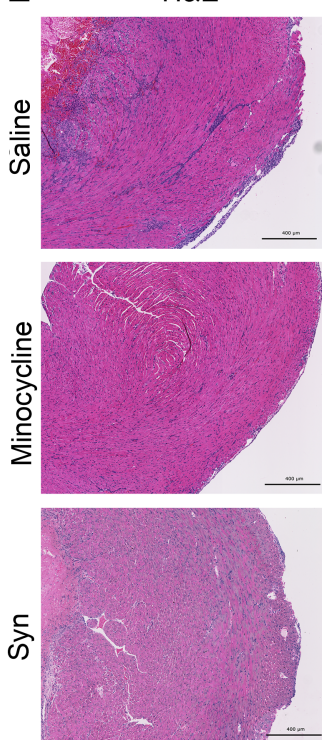

CD3

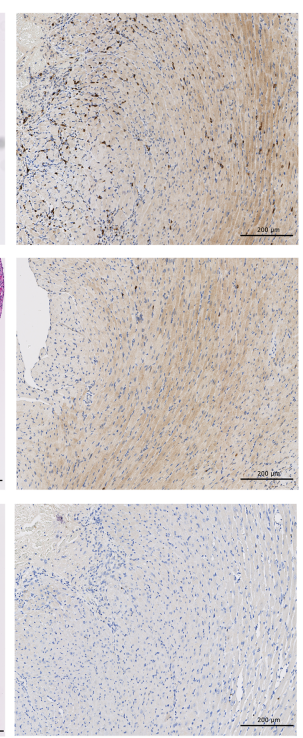

C

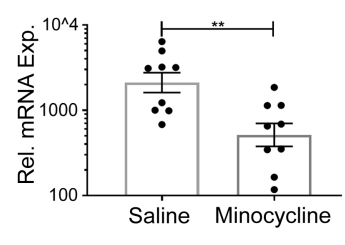

TNF $\alpha$

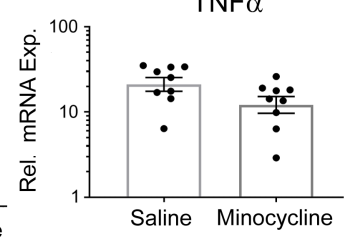

IL2

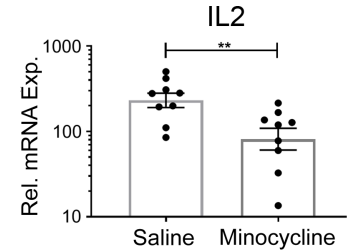

IFN $\gamma$



Figure 7. Minocycline treatment reduced the inflammatory profile of AR in a murine heterotopic heart transplant model. (A) $C 57 \mathrm{BL} / 6$ mice were transplanted with hearts from BALB/c mice, representing a full MHC mismatch. Recipient mice received intraperitoneal injections of minocycline ( $n$ $=9)$ or saline $(n=9)$ twice daily starting 36 hours before the allogeneic cardiac transplant, through to postoperative day 4 (POD4) and euthanized; all allografts were still viable (with palpable beat) at this time. Four syngeneic cardiac transplants (C57BL/6 donors to C57BL/6 recipients, no treatment) were used as controls. (B and C) The mRNA transcript expression (Rel. mRNA Exp., presented on a log scale) was measured by real-time PCR. Four of the $14 \mathrm{KDGs}$, including the top-ranked key driver, Caspase-1, were significantly reduced in the allografts of the minocycline-treated animals compared with saline-treated animals. Significant attenuation of allograft mRNA expression of several proinflammatory chemokines and cytokines with minocycline treatment was observed most prominently with IFN- $\gamma$ and IL-2; TNF- $\alpha$ expression was not significantly different. (D) Protein concentrations of IFN- $\gamma$ and TNF- $\alpha$ (Prot. conc. $\mathrm{pg} / \mathrm{mL}$ ) in sera were measured with array beads, and the results were in line with the allograft expression of the corresponding transcripts. (E and F) Representative H\&E and immunohistochemistry staining for CD3 (T cells) and galectin-3 (macrophages) (both stained brown) in cardiac allografts demonstrated substantially less inflammatory cell infiltrate in the minocycline-treated animals compared with those given saline ( $n=9$ in each group), which was quantified by the Positive Pixel Count Algorithm (Pos. Pix. Ct/mm2, Version 9 , Leica Biosystems). Furthermore, infiltration of both cell types in the allogeneic allografts of minocycline-treated mice was not significantly different from that in the syngeneic grafts $(n=3)$. Relative expression of mRNA transcripts was calculated using the $\Delta \Delta C T$ method, normalized to the expression in the syngeneic transplant group, with GAPDH as the housekeeping gene. Scale bars: $400 \mu \mathrm{m}$ (H\&E and CD3), $200 \mu \mathrm{m}$ (galectin-3). For mRNA and serum protein data, Student's 2-tailed $t$ test was used to analyze differences between the 2 groups for normally distributed data, and the Mann-Whitney $U$ test was used for non-normally distributed data. Cell infiltrate quantification was compared using 1-way ANOVA and Tukey's multiple comparisons test. All data are presented as mean \pm SEM $\left({ }^{*} P<0.05,{ }^{* *} P<0.01,{ }^{* *} P<0.001\right)$. IP, intraperitoneal; POD, postoperative day; minoc, minocycline-treated allografts; saline, saline-treated allografts; syn, syngeneic grafts.

the time of harvesting on POD14 ( $n=4$ each group). Troponin I staining to assess viable myocardium showed significantly more staining in the minocycline-treated group $(P=0.020)$ (Figure $8 \mathrm{~B})$. Next, animals received minocycline or saline through to POD27 (Figure 8C); allograft survival was assessed 
A

Start minocycline/saline (36 hrs pretransplantation) IP twice daily

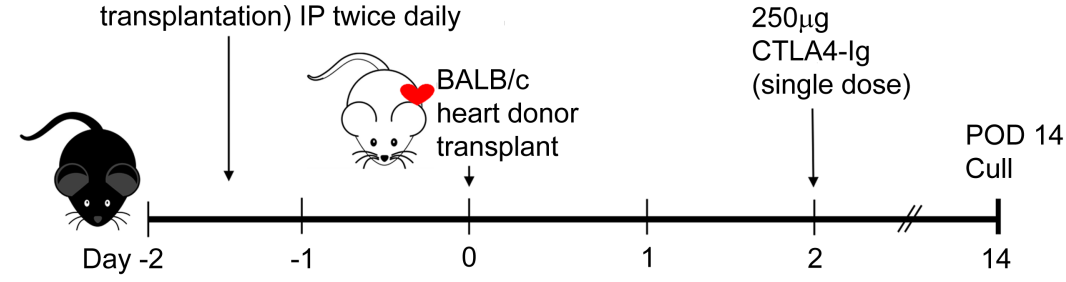

B
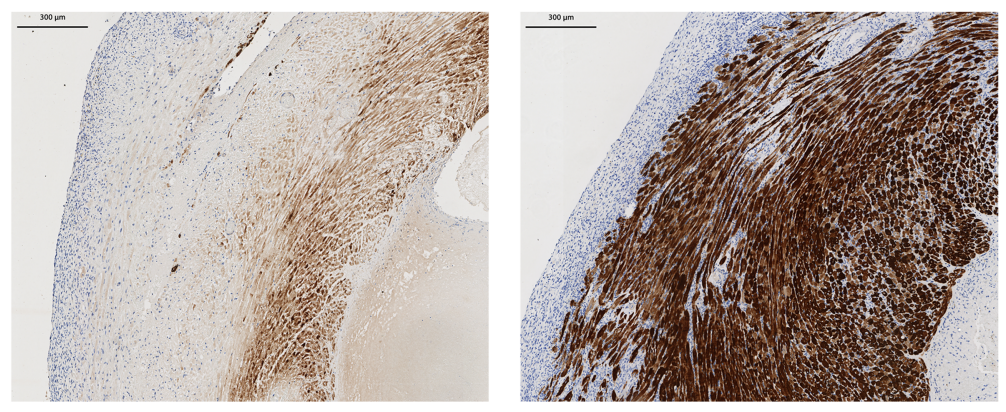

Stop minocycline/saline

C Start minocycline/saline (36 hrs pretransplantation) IP twice daily

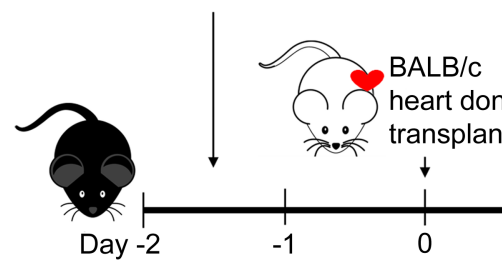
$250 \mu \mathrm{g}$ CTLA4-Ig (single dose)

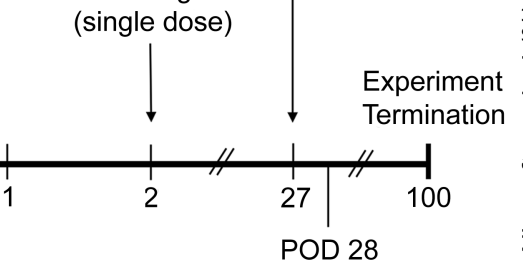

assessment of graft survival

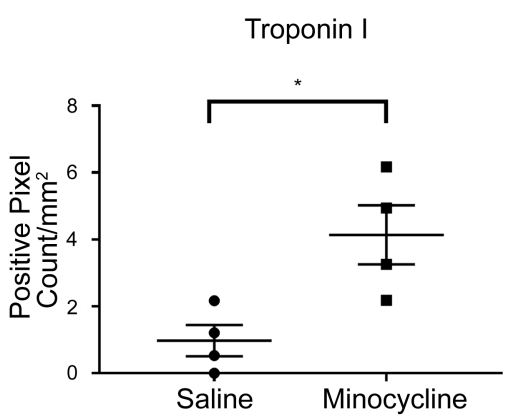

D

Allograft survival POD 28 (censored for non-rejection events)



Figure 8. Minocycline as adjunct to immunosuppression confers allograft survival benefit during treatment period. (A) Minocycline and saline were compared as adjunct agents to immunosuppression, administered as a single low dose of CTLA4-Ig (250 $\mu$ g) IP on POD2. First, animals were given minocycline or saline ( $n=4$ in each group) through to POD14 and culled; all allografts were still viable at the time. (B) Representative immunohistochemistry of Troponin I (brown staining) of POD14 allograft myocardium treated with CTLA4-Ig with adjunct saline, versus CTLA4Ig with adjunct minocycline, respectively. Quantification using the Positive Pixel Count Algorithm (Version 9, Leica Biosystems) demonstrated significantly greater preservation of Troponin I staining of cardiomyocytes in the minocycline group, indicating less myocardial necrosis compared with the saline group. Scale bar: $300 \mu \mathrm{m}$. (C) Next, the effect of adjunct minocycline or adjunct saline treatment to single-dose CTLA4-lg on allograft survival was compared, with animals receiving minocycline $(n=8)$ or saline $(n=11)$ while grafts remained viable through to POD27, after which treatment was ceased. Graft survival analysis was performed at POD28. Animals were observed thereafter without further intervention and euthanized when allografts completely rejected or when the end of the experimental period (POD100) was reached. (D) Evaluation of allograft survival at POD28 (censored for nonrejection events) immediately following the cessation of adjunct minocycline/saline treatment demonstrated superior graft survival in the minocycline group ( 0 out of 7 grafts lost) compared with the saline group ( 7 out of 11 grafts lost) $(P=0.007)$. Student's 2-tailed $t$ test was used for the comparison of IHC staining of Troponin I in the POD14 cardiac allografts between the 2 treatment groups $\left({ }^{*} P<0.05\right)$. Kaplan-Meier survival analysis using the log-rank test was applied to compare allograft survival at POD28. CTLA4-Ig, cytotoxic T lymphocyte-associated protein 4-immunoglobulin.

at POD28, but animals were not euthanized if their grafts were still viable. Significant graft survival benefit from the additional minocycline was observed $(P=0.007)$ (Figure 8D).

Mice that received CTLA4-Ig plus minocycline/saline through to POD27 were allowed to go out to day 100 , when the graft survival advantage was lost $(P=0.107)$, suggesting that minocycline's efficacy was as an additional immunosuppressive agent but was not inducing tolerance or any protective effect that outlasted its use (Supplemental Figure 8).

In summary, with ongoing treatment, minocycline, as an adjunct to CTLA4-Ig immunosuppression, extended cardiac allograft survival. 


\section{Discussion}

This study shows the derivation of KDGs among dysregulated AR pathways in renal transplantation, using human allograft microarray data from publicly available data sets and our AR pathways in renal transplantation, using human allograft microarray data from publicly available data sets and our GoCAR study cohort (26). These molecules served as targets for therapeutic discovery, demonstrated by the identification of minocycline by drug-repositioning strategies and in vivo demonstration of its biological efficacy.

An important aspect of this meta-analysis of patient samples is that these rejection episodes occur in the presence of CNI-based maintenance immunosuppression, suggesting that the molecular pathways identified here are those that are not affected by or escape the current immune-modulating effect of standard therapy. In the setting of renal transplantation, few meta-analysis studies of transcriptomic data have been undertaken, and these identified DEGs in AR in single-organ and cross-organ data sets (34), allograft fibrosis (25), and tolerance (49). Extending upon these studies, in this study we have constructed gene regulatory networks among the DEGs and further incorporated genotype data to predict the key drivers within the gene modules.

Specific immune response processes represented by the upregulated meta-genes included cell activation and antigen presentation, and these are highly expressed in innate cell types, particularly macrophages, dendritic cells, and monocytes. This is consistent with recognition over the last 2 decades that innate immune responses play a key role in the development and propagation of AR (50), which can occur even in the absence of $\mathrm{T}$ cells (51). It remains, however, that current standard immunosuppression regimens are directed at suppressing $\mathrm{T}$ cell activation and proliferation, with no agents targeting the monocyte/macrophage lineage. Macrophages have been demonstrated to accumulate in the renal allograft in the clinical setting in association with both TCMR and ABMR and correlate with rejection severity and graft survival $(52,53)$. Recently their role has been included as immunopathological evidence of ABMR in cardiac transplantation using macrophage presence in capillaries (54). In our meta-analyses, each of the included studies reported the majority of patients were receiving CNI-based therapy, and while not specifically presented here, we found that IL-2 transcripts were low and nondifferentially expressed across AR and non-AR samples, supporting that this pathway of $\mathrm{T}$ cell activation was adequately suppressed. We also observed that enrichment of B cell transcripts was absent despite the inclusion of ABMR samples, consistent with previous studies showing no differential expression of B cell-associated transcripts between ABMR and non-ABMR biopsies $(55,56)$. Our findings lend further support to the need for therapeutic strategies to target the pathways responsible for monocyte/ macrophage lineage activation for $\operatorname{AR}$ prevention $(57,58)$.

Of the $14 \mathrm{KDG}$ s derived, a few have well-described functions in the immune response (e.g., CASP1, HCK, VAV1), while others have not been as well characterized (e.g., EVI2A) and represent opportunities for future study. Published literature for the more characterized key drivers provides support of their identification as key mediators in dysregulated immunopathological pathways of AR. The top-ranked KDG, CASP1, encodes a protease that cleaves pro-IL-1 $\beta$ and IL-18 into their active forms as potent proinflammatory cytokines (59). CASP1 is a component of the inflammasome complexes, which are key innate response elements to pathogen-associated molecular patterns or damage-associated molecular patterns; the latter are triggers for allograft rejection (60). In the setting of AR, selective CASP1 inhibition using an experimental compound to modulate the inflammasome complex was recently described in a rodent liver transplant model (61). Disruption of the activity of VAV1, a signal transducer with a central role in the hematopoietic system, has been shown to prolong murine cardiac allograft survival (62). It is a known substrate of the family of Src-related tyrosine kinases (SFKs), which includes HCK, a KDG belonging to the same module. Consistent with this, our computational analyses placed VAV1 as a downstream molecule to HCK. Members of the SFKs are known regulators of immune response signaling pathways, and in rejecting murine cardiac allografts, HCK has been shown to exhibit the greatest increase in expression and activity of the SFKs (63). A number of FDA-approved Src inhibitors, which are therapeutics in a number of cancer types, were identified in our DrugBank search as inhibitors of VAV1 or HCK. This included dasatinib. Beyond demonstration that it prolongs murine graft survival, our group has shown that it suppresses HCK expression and attenuates renal fibrosis development in vivo (64), and this may occur by it limiting inflammation. PSMB10 and PSME1 are members of the same module that encode for a catalytic subunit and a regulator component of the immunoproteasome, a specialized form of the multi-subunit proteasome with enhanced capability for antigen processing. Bortezomib, a nonselective proteasome inhibitor used in myeloma, has been evaluated in clinical trials of ABMR (65) but 
was limited by unacceptable toxicity; more selective proteasome inhibitors are needed. Another of the KDGs, PLEK, encodes a protein substrate of protein kinase C (PKC). Sotrastaurin, a novel small-molecule inhibitor of PKC, was previously developed for prophylaxis of AR (66) but did not progress beyond phase II clinical trials. Nevertheless, the data on the KDGs and their associated pathways in AR support the discovery of the KDGs as relevant therapeutic targets to prevent rejection.

While KDG overexpression was demonstrated in additional clinical kidney and heart transplant biopsy data sets, this was not observed in liver and lung transplant data sets of AR, suggesting that the KDGs may not be important regulators in these organ types. Immunologically, the liver and lung have a heightened innate immune defense system because of their constant exposure to antigens from the gastrointestinal tract and the external environment, respectively. Even in the steady-state environment, the liver has an abundance of tissue-resident macrophages (67), and in the lungs, macrophages comprise approximately $95 \%$ of airway leukocytes (68). This is in contrast to kidneys and heart tissue, which could explain the differences observed. While there are undoubtedly DEGs in AR that are common to all organ types (34), including downstream effector molecules such as inflammatory cytokines that shape the AR phenotype, initiating and/or central regulatory molecules in dominant biological processes may be organ specific.

Our study demonstrated the feasibility of an in silico approach to identify drugs for repurposing in AR, which has been described by only 1 other study in this setting (29). While CMAP was used in our study to predict drugs that could inhibit the key driver-based gene set, we also hypothesized that there are valid reasons for why established immunosuppressive agents in clinical use in renal transplantation were not demonstrated to have significant negative correlations with our input AR gene set. Because patients within the cohorts of the meta-analyses were primarily receiving CNI-based therapy already, these agents were not expected to rank highly against our input gene set. Indeed, the CNI tacrolimus generated a connectivity score of -0.06 with a $P$ value of 0.28 (data not shown), strengthening our confidence in the predictive capacity of CMAP in this study.

Minocycline was chosen for experimental validation because it was the only agent discovered in both repositioning strategies. Supporting its biological plausibility as a potential therapeutic in AR, its described antiinflammatory and immunomodulatory properties has led to its repurposing into clinical trials of nonmicrobial conditions, including a recent multicenter prospective randomized controlled trial that compared minocycline with placebo on risk of conversion from a clinically isolated syndrome to multiple sclerosis, an immune-mediated inflammatory CNS disease (69). Minocycline also has an acceptable side effect profile and has a convenient twice-daily oral dosing regimen in humans, which are relevant considerations in clinical transplantation. Beyond its inhibitory effects on the inflammasome/CASP1 pathway $(45,70,71)$, minocycline may also suppress monocyte and macrophage activation and functions by other mechanisms to attenuate inflammatory responses $(72,73)$. Modulation of macrophage polarization from the M1 (proinflammatory) phenotype that drives injury, toward the M2 (antiinflammatory) phenotype by minocycline treatment has also been reported in preclinical studies (74). Although the impact on T cells is thought to be downstream of the monocyte-directed effects of minocycline, limited studies have demonstrated there may also be direct effects on T cell priming $(75,76)$. Our data would be consistent with this because there was reduced IFN- $\gamma$ gene expression within the grafts of mice treated with minocycline, which correlated with reduced serum protein levels of IFN- $\gamma$ in these same mice.

Given the absence of therapeutics directed at the monocyte/macrophage lineage for AR prophylaxis in renal transplantation, minocycline could provide additional and nonredundant immunosuppression. In our studies, minocycline with CTLA4-Ig prolonged allograft survival during the treatment period, but this effect did not extend after withdrawal of minocycline. This suggests that minocycline is useful in limiting inflammation but does not promote tolerance and would require ongoing administration. Because the half-life of both oral and parenteral minocycline in a rodent approximates 2-3 hours only (77), in contrast to humans where the half-life is 12 to 18 hours (78), the twice-daily dosing regimen in our in vivo model may not have resulted in its maximal efficacy. However, given its much longer half-life in humans, the optimal dosing of minocycline in its established clinical applications is twice daily, the same as that of the transplant immunosuppressive agents tacrolimus and mycophenolate, which have similar half-lives. Nevertheless, the significant reduction in macrophage infiltration at POD4 with minocycline treatment is encouraging, supporting further evaluation in a clinical trial as an adjunctive therapy to standard $\mathrm{T}$ cell-oriented immunosuppression for AR prevention. This repurposing is also favored by its current generic status, its low cost, and its acceptable side effect profile. 
Given its additive role in blocking alternative activation immune pathways, it may allow lower immunosuppression with standard agents.

There are some limitations to our study. Methodologically, due to a paucity of SNP data in public databases, the directional Bayesian network within the meta-network module and the key driver predictions were built on the GoCAR genotype data alone and will require confirmation in future studies. Second, our meta-analysis incorporated both clinical cases of AR (identified on indication biopsies) and subclinical cases (AR identified on protocol biopsies), and the significance of subclinical rejection with subsequent graft loss has been the subject of some debate. However, our group has shown in a multicenter prospective GoCAR cohort that allografts with subclinical rejection at 3 months posttransplant have reduced allograft survival (26), and studies that evaluated the gene expression profile of biopsies with clinical AR and subclinical AR suggest the 2 entities represent a continuum of alloimmune activation and are not distinct $(79,80)$. Our findings here support these observations, with significant correlation of KDG expression with subsequent graft loss in both our GoCAR data set comprising protocol biopsies samples and the external validation data set comprising indication biopsies. Third, we selected only 1 agent for biological validation, and it is possible that other therapeutics discovered in CMAP could be more effective in the AR setting. Finally, beyond the known inhibitory effects of minocycline on CASP1 expression and activation, the mechanisms by which it directly and/or indirectly alters expression of other KDG warrants attention in future experimental studies.

The dominance of innate immune cell transcripts among the overexpressed genes in AR confirm the deficiencies of standard regimens that are mainly directed at inhibiting $\mathrm{T}$ cell responses. Therapeutic manipulation of KDGs among these dysregulated innate pathways has the potential for meaningful clinical impact. Prioritization of these as targets for therapeutic intervention facilitated the computational discovery of drug candidates for repurposing, and experimental validation of a select candidate was demonstrated. Taken together, our results show that integrative bioinformatic methods can provide opportunities for meaningful molecular target and drug discovery in the niche field of solid organ transplantation. This can guide the selection of nonredundant drugs for clinical trials in AR prevention, with the ultimate goal of extending graft survival.

\section{Methods}

A detailed description of the bioinformatic methods is provided in Supplemental Methods.

Data set description. The 10 public microarray data sets of kidney biopsies posttransplant were collected from the public domain. A further 2 data sets belonged to our research group as part of the Genomics of Chronic Allograft Dysfunction (GoCAR) study, 1 of which is published, and both are available via GEO. Data sets were randomly divided into discovery ( 7 data sets, Supplemental Table 1$)$ and validation (5 data sets, Supplemental Table 1) cohorts (26-36). Samples were dichotomized to AR (this included TCMR and borderline TCMR, ABMR, and mixed cases) or non-AR, as identified by their original studies. The majority of patients in each of these data sets were under CNI-based immunosuppression, as described in their relevant publications. The discovery sets were used to identify the differentially expressed meta-signatures in $\mathrm{AR}$ versus non-AR patients and the KDGs associated with AR from a Bayesian network constructed using the expression correlation network of meta-gene signatures and the genotype data of the GoCAR cohort. The independent kidney allograft data sets were used to validate the association of KDGs with AR. In addition, the expression of KDGs was evaluated in nonkidney transplant gene expression data sets (3 heart, 2 lung, and 2 liver data sets) (Supplemental Table 1) (37-42).

Bioinformatic analysis for meta-signature, key driver identification, and drug repurposing. A detailed description of bioinformatic analysis of identification of meta-gene signature, key drivers of $A R$, and subsequent discovery of repurposed drugs targeting key drivers is provided in Supplemental Methods and depicted in Supplemental Figure 1. Briefly, a meta-analysis first identified DEGs across multiple microarray data sets by summarizing the effect size of between AR and non-AR groups considering both the expression value difference of 2 groups and sample size. To strengthen the results, we used another approach by combining $P$ values from each data set with limma test into meta- $P$ values. By combining effect size and meta- $P$ approaches, meta-gene signatures were identified based on the criteria described in Supplemental Methods and then subjected to GO and immune cell type enrichment analysis.

Next, a meta-expression correlation network was built with expression correlation coefficients (meta- $r$ ) among the meta-genes across the data sets and then divided into submodules using the MCL algorithm. The modules associated with AR were further identified by comparing the connectivity of module genes in AR samples with non-AR samples. Differentially connected modules were then applied to construct the 
directed regulatory network using a Bayesian network model $(81,82)$, with eQTL SNP data derived from our GOCAR data as a prior. The KDGs in each module were algorithmically derived based on the number of out-degrees and number of downstream genes, and the algorithm has been previously described (15). Cox regression model was applied to correlate expression of KDGs with graft loss, and prediction capability of graft loss with KDGs was estimated in 2 data sets (GSE21374 and our GoCAR 3-month data set).

Last, the key driver-based signature of AR was used to query CMAP to identify potential therapeutic drugs that can induce an opposing effect on the expression of AR signatures by following the approach described previously $(25,83)$. These potential drugs were further queried against DrugBank database (22) to prioritize the drugs that directly target 1 or more KDGs as the candidate drug for experimental validation.

Animals. BALB/c and C57BL/6 male WT mice ( $~ 8$ weeks of age) were purchased from the Australian Animal Research Centre. Animals were 9-12 weeks of age at the time the experimental procedures were undertaken. Mice were kept in the Westmead Hospital Animal Care Facility in pathogen-free conditions, on a 12-hour light/12-hour dark cycle.

In vivo rodent transplant model of $A R$. A murine abdominal heterotopic cardiac transplant model of AR was used as described previously (84). The survival of heart allografts with this strain combination is between 7 and 9 days without intervention (85).

$\mathrm{BALB} / \mathrm{c}$ WT male hearts were transplanted into the abdomen of C57BL/6 mice (day 0) as the allogeneic transplants; C57BL/6 hearts were transplanted into C57BL/6 mice as the syngeneic transplants. Cessation of allograft beat within the first 72 hours of surgery was defined as technical failure, and these animals were excluded from the experiments.

Transplanted recipients that were in the minocycline treatment groups across all the experimental arms received minocycline (M9511, MilliporeSigma) twice daily at $50 \mathrm{mg} / \mathrm{kg}$ per dose, administered through the IP route; saline treatment (sham) groups received Dulbecco's phosphate-buffered saline (Lonza Biologics) twice daily, which was also corrected to body weight. As the aim of this study was to demonstrate any potential effect of minocycline, the cumulative daily dose chosen was at the upper limit of that tolerated in published rodent studies $(86,87)$. Animals in the saline and minocycline groups received this treatment starting 36 hours before transplantation, similar to experimental rodent models of organ ischemia/reperfusion injury (88). For the experiments with immunosuppression, a single 250- $\mu \mathrm{g}$ IP dose of CTLA4-Ig (Walter and Eliza Hall Institute of Medical Research Antibody Facility) was given. Its use as a single reduced dose at day 2 has been described in rodent models previously in the study of allograft immune processes and prolonged graft survival without inducing tolerance $(89,90)$.

Grafts were evaluated daily by transabdominal palpation of heartbeat by 2 observers, 1 of whom was blinded to the experimental group of each animal. Complete rejection was defined as cessation of palpable heartbeat of the allograft (91), confirmed upon visual inspection under sedation before euthanasia by exsanguination.

Experimental arms. Treatment with minocycline alone and saline alone was first compared. Animals received either minocycline alone $(n=9) 50 \mathrm{mg} / \mathrm{kg}$ IP twice daily or saline alone $(n=9)$ IP twice daily through to POD4; syngeneic animals $(n=4)$ were used as controls. All animals were euthanized at POD4; all grafts were still viable. Next, impact on allograft survival was compared between minocycline treatment alone compared with saline treatment but administered until allografts completely rejected.

The impact of minocycline versus saline, when used as adjunct treatment to immunosuppression, was evaluated. This was assessed at POD14 when allografts were still viable. All animals received a single IP dose of $250 \mu \mathrm{g}$ CTLA4-Ig on POD2 as immunosuppression and either minocycline $(n=4)$ or saline $(n=4)$ as described through to POD14. Animals were euthanized at POD14 to allow comparison of allograft histology. Finally, impact on allograft survival was examined. All animals received a single IP dose of CTLA4-Ig on POD2, and either minocycline $(n=8)$ or saline $(n=11)$ up to and including POD27, after which minocycline/ saline treatment was ceased and the animals observed through to POD100, the end of the experimental period.

$R N A$ extraction from allografts and quantitative real-time $P C R$. mRNA from allograft tissue was extracted using RNeasy Mini Kit (QIAGEN). Two micrograms of extracted RNA from the murine cardiac allografts was used for conversion to cDNA, using the SuperScript III First-Strand Synthesis Kit (Thermo Fisher Scientific) with oligo(dT) primers, as per the manufacturer's instructions. Quantitative real-time PCR was performed using TaqMan gene expression assays (IDs in Supplemental Methods and Supplemental Table 6) (Thermo Fisher Scientific) and Platinum Quantitative PCR SuperMix-UDG (Thermo Fisher Scientific) on the Bio-Rad Touch CFX 384 system. Samples were run in duplicate, and GAPDH was used as the housekeeping gene. 
Cytometric bead array for protein quantification in sera. LEGENDplex Mouse Inflammation Panel (13-plex) (BioLegend, California, USA) was used to quantify the 2 cytokines of interest in mouse serum: IFN- $\gamma$ and TNF- $\alpha$. Proteins were detected on BD FACSCanto II (BD Biosciences) following the manufacturer's instructions (BioLegend). Data were analyzed using BioLegend's LEGENDplex Data Analysis Software.

Histology and immunohistochemistry. H\&E staining of allograft tissue cross sections from the 4-day and 14-day models on formalin-fixed, paraffin-embedded sections was performed. Macrophages were detected using primary rat anti-mouse galectin-3 antibody (clone M3/38) at 1:8000 dilution (Cedarlane), then secondary antibody rabbit anti-rat IgG (BA-4001) at a 1:200 dilution (Vector Laboratories). $\mathrm{T}$ cells were detected using primary rabbit anti-mouse CD3 antibody (ab5690) at 1:100 dilution (Abcam). Troponin I staining to assess for allograft myocyte necrosis in the day 14 model was detected using primary rabbit anti-mouse Troponin I antibody (ab47003) at 1:100 dilution (Abcam). All IHC staining was performed using Leica BOND RX Automated Research Stainer (Leica Biosystems, Germany) and the BOND Polymer Refine Detection Kit (Leica Biosystems, United Kingdom). CD3- and galectin-3-stained images were scanned and analyzed using Aperio ImageScope (version 12.4.0; Leica Biosystems, USA) and the Positive Pixel Count Algorithm (Version 9; Leica Biosystems), as described in Supplemental Methods.

Statistics. Prism software version 7.0 (GraphPad Software) was used for PCR data analysis and serum protein analysis, staining quantification, survival analysis, and graphical presentation of results.

For real-time PCR data of the POD4 experiments, the $\triangle \Delta C T$ method was applied to calculate the level of comparative expression of each gene transcript for each animal in the allogeneic saline and minocycline experimental groups relative to the syngeneic control group, normalized to GAPDH as the housekeeping gene. Data were log transformed, then analyzed for outliers using Grubb's test; identified outliers were excluded from analysis. Normality of distribution was assessed using Shapiro-Wilk test. Student's 2-tailed $t$ test was used to compare the relative expression between the saline and minocycline treatment groups for normally distributed data; Mann-Whitney $U$ test was used for non-normally distributed data. Results were expressed as mean \pm SEM. For IHC staining of CD3 and galectin-3, 1-way ANOVA with Tukey's multiple comparisons test were performed to analyze the differences between the 3 experimental groups in the POD4 model; Student's $t$ test was used to compare Troponin I staining between the 2 groups in the POD14 model. All results are presented as the mean \pm SEM. Statistical significance was defined at a $P$ value of less than 0.05 .

For protein quantification in the sera, data were analyzed in the same manner as the PCR data except log transformation was not undertaken.

For allograft survival data, the log-rank test was used for evaluation at POD28 and POD100 with a significance level at $P<0.05$.

Study approval. The GoCAR study was approved by the institutional review boards of the participating institutions as described previously (26). Written informed consent was obtained from all enrolled patients. All experimental procedures using animals were performed with the approval of the Animal Ethics Committee of Western Sydney Local Health District (Westmead Institute for Medical Research) and adhered to the guidelines set by the National Health and Medical Research Council of Australia.

\section{Author contributions}

ZY performed the data set search and bioinformatic analyses and contributed to the writing of the paper. KLK designed and performed the animal experiments and related work and data analyses and wrote the paper. LL conducted the drug-repositioning analyses. MH assisted with animal experiments and related work. BL performed the animal surgeries. LN conducted the histology imaging analyses. EJV performed the bead array experiment. MCM and CW assisted with data analyses. SA assisted with data analyses and edited the paper. BM was involved in study conception and data analyses and edited the paper. PJO participated in design of animal experiments and the related work and in data analyses and edited the paper. WZ conceptualized the study, designed the bioinformatic analyses, and edited the paper.

\section{Acknowledgments}

Thank you to Jonathan Erlich (Department of Nephrology, Prince of Wales Hospital) for providing advice on the animal experimental design and Yi Vee Chew, Heather Burns, and Ahmer Hameed (Westmead Institute for Medical Research) for assistance with animal experiments. 
Address correspondence to: Weijia Zhang or Barbara Murphy, Department of Medicine, Icahn School of Medicine at Mount Sinai, 1 Gustave L Levy Place, Box 1243, New York, New York 10029, USA. Phone: 212.241.2883; Email: weijia.zhang@mssm.edu (WZ). Phone: 212.241.8001; Email: barbara.murphy@ mssm.edu (BM). Or to: Philip J. O'Connell, Westmead Institute for Medical Research, University of Sydney, 176 Hawkesbury Rd., Westmead, New South Wales 2145, Australia. Phone: 61.286273000; Email: philip.oconnell@sydney.edu.au.

1. Girlanda R, et al. Monocyte infiltration and kidney allograft dysfunction during acute rejection. Am J Transplant. 2008;8(3):600-607.

2. Haas M, et al. The Banff 2017 Kidney Meeting Report: Revised diagnostic criteria for chronic active T cell-mediated rejection, antibody-mediated rejection, and prospects for integrative endpoints for next-generation clinical trials. Am J Transplant. 2018;18(2):293-307.

3. Opelz G, Döhler B, Collaborative Transplant Study Report. Influence of time of rejection on long-term graft survival in renal transplantation. Transplantation. 2008;85(5):661-666.

4. Moreso F, et al. Subclinical rejection associated with chronic allograft nephropathy in protocol biopsies as a risk factor for late graft loss. Am J Transplant. 2006;6(4):747-752.

5. Gago M, Cornell LD, Kremers WK, Stegall MD, Cosio FG. Kidney allograft inflammation and fibrosis, causes and consequences. Am J Transplant. 2012;12(5):1199-1207.

6. Reeve J, et al. Diagnosing rejection in renal transplants: a comparison of molecular- and histopathology-based approaches. Am J Transplant. 2009;9(8):1802-1810.

7. Halloran PF, Venner JM, Famulski KS. Comprehensive analysis of transcript changes associated with allograft rejection: combining universal and selective features. Am J Transplant. 2017;17(7):1754-1769.

8. Menon MC, Keung KL, Murphy B, O'Connell PJ. The use of genomics and pathway analysis in our understanding and prediction of clinical renal transplant injury. Transplantation. 2016;100(7):1405-1414.

9. Waldron L, Riester M. Meta-Analysis in gene expression studies. In: Mathé E, Davis S, eds. Statistical Genomics: Methods and Protocols. Springer New York; 2016:161-176.

10. Tseng GC, Ghosh D, Feingold E. Comprehensive literature review and statistical considerations for microarray meta-analysis. Nucleic Acids Res. 2012;40(9):3785-3799.

11. Stuart JM, Segal E, Koller D, Kim SK. A gene-coexpression network for global discovery of conserved genetic modules. Science. 2003;302(5643):249-255.

12. Allocco DJ, Kohane IS, Butte AJ. Quantifying the relationship between co-expression, co-regulation and gene function. $B M C$ Bioinformatics. 2004;5:18.

13. Schadt EE, et al. An integrative genomics approach to infer causal associations between gene expression and disease. Nat Genet. 2005;37(7):710-717.

14. Zhang BZ, Zhu J. Identification of key causal regulators in gene networks. Lecture Notes in Engineering and Computer Science. 2013;2:1309-1312.

15. Zhang B, et al. Integrated systems approach identifies genetic nodes and networks in late-onset Alzheimer's disease. Cell. 2013;153(3):707-720.

16. Peters LA, et al. A functional genomics predictive network model identifies regulators of inflammatory bowel disease. Nat Genet. 2017;49(10):1437-1449.

17. Mäkinen VP, et al. Integrative genomics reveals novel molecular pathways and gene networks for coronary artery disease. PLoS Genet. 2014;10(7):e1004502.

18. Langone A, Sanders M. Drugs in development for prophylaxis of rejection in kidney-transplant recipients. Transpl Res Risk Manag. 2015;7:59-69.

19. Pushpakom S, et al. Drug repurposing: progress, challenges and recommendations. Nat Rev Drug Discov. 2019;18(1):41-58.

20. Sawada R, Iwata M, Tabei Y, Yamato H, Yamanishi Y. Predicting inhibitory and activatory drug targets by chemically and genetically perturbed transcriptome signatures. Sci Rep. 2018;8(1):156.

21. Gaulton A, et al. ChEMBL: a large-scale bioactivity database for drug discovery. Nucleic Acids Res. 2012;40(Database issue):D1100-D1107.

22. Wishart DS, et al. DrugBank: a knowledgebase for drugs, drug actions and drug targets. Nucleic Acids Res. 2008;36(Database issue):D901-D906.

23. Lamb J, et al. The Connectivity Map: using gene-expression signatures to connect small molecules, genes, and disease. Science 2006;313(5795):1929-1935.

24. Subramanian A, et al. A next generation connectivity map: L1000 platform and the first 1,000,000 profiles. Cell. 2017;171(6):1437-1452.e17.

25. Li L, et al. Novel therapeutics identification for fibrosis in renal allograft using integrative informatics approach. Sci Rep. 2017;7:39487.

26. O'Connell PJ, et al. Biopsy transcriptome expression profiling to identify kidney transplants at risk of chronic injury: a multicentre, prospective study. Lancet. 2016;388(10048):983-993.

27. Flechner SM, et al. Kidney transplant rejection and tissue injury by gene profiling of biopsies and peripheral blood lymphocytes. Am J Transplant. 2004;4(9):1475-1489.

28. Saint-Mezard P, et al. Analysis of independent microarray datasets of renal biopsies identifies a robust transcript signature of acute allograft rejection. Transpl Int. 2009;22(3):293-302.

29. Roedder S, Kimura N, Okamura H, Hsieh SC, Gong Y, Sarwal MM. Significance and suppression of redundant IL17 responses in acute allograft rejection by bioinformatics based drug repositioning of fenofibrate. PLoS One. 2013;8(2):e56657.

30. Reeve J, et al. Molecular diagnosis of T cell-mediated rejection in human kidney transplant biopsies. Am J Transplant. 
2013;13(3):645-655.

31. Ó Broin P, et al. A pathogenesis-based transcript signature in donor-specific antibody-positive kidney transplant patients with normal biopsies. Genom Data. 2014;2:357-360.

32. Einecke G, et al. A molecular classifier for predicting future graft loss in late kidney transplant biopsies. J Clin Invest. 2010;120(6):1862-1872.

33. Halloran PF, et al. Potential impact of microarray diagnosis of T cell-mediated rejection in kidney transplants: the INTERCOM study. Am J Transplant. 2013;13(9):2352-2363.

34. Khatri P, et al. A common rejection module (CRM) for acute rejection across multiple organs identifies novel therapeutics for organ transplantation. J Exp Med. 2013;210(11):2205-2221.

35. Ling XB, et al. Integrative urinary peptidomics in renal transplantation identifies biomarkers for acute rejection. $J$ Am Soc Nephrol. 2010;21(4):646-653.

36. Naesens M, et al. Progressive histological damage in renal allografts is associated with expression of innate and adaptive immunity genes. Kidney Int. 2011;80(12):1364-1376.

37. Morgun A, et al. Molecular profiling improves diagnoses of rejection and infection in transplanted organs. Circ Res. 2006;98(12):e74-e83.

38. Holweg CT, et al. Identification and classification of acute cardiac rejection by intragraft transcriptional profiling. Circulation. 2011;123(20):2236-2243.

39. Patil J, Lande JD, Li N, Berryman TR, King RA, Hertz MI. Bronchoalveolar lavage cell gene expression in acute lung rejection: development of a diagnostic classifier. Transplantation. 2008;85(2):224-231.

40. Gimino VJ, Lande JD, Berryman TR, King RA, Hertz MI. Gene expression profiling of bronchoalveolar lavage cells in acute lung rejection. Am J Respir Crit Care Med. 2003;168(10):1237-1242.

41. Bohne F, et al. Intra-graft expression of genes involved in iron homeostasis predicts the development of operational tolerance in human liver transplantation. J Clin Invest. 2012;122(1):368-382.

42. Asaoka T, et al. Differential transcriptome patterns for acute cellular rejection in recipients with recurrent hepatitis $\mathrm{C}$ after liver transplantation. Liver Transpl. 2009;15(12):1738-1749.

43. Brundula V, Rewcastle NB, Metz LM, Bernard CC, Yong VW. Targeting leukocyte MMPs and transmigration: minocycline as a potential therapy for multiple sclerosis. Brain. 2002;125(pt 6):1297-1308.

44. Garrido-Mesa N, et al. The intestinal anti-inflammatory effect of minocycline in experimental colitis involves both its immunomodulatory and antimicrobial properties. Pharmacol Res. 2011;63(4):308-319.

45. Shahzad K, et al. Stabilization of endogenous Nrf2 by minocycline protects against Nlrp3-inflammasome induced diabetic nephropathy. Sci Rep. 2016;6:34228.

46. Theruvath TP, et al. Minocycline and N-methyl-4-isoleucine cyclosporin (NIM811) mitigate storage/reperfusion injury after rat liver transplantation through suppression of the mitochondrial permeability transition. Hepatology. 2008;47(1):236-246.

47. Versluis DJ, ten Kate FJ, Wenting GJ, Weimar W. Mononuclear cells infiltrating kidney allografts in the absence of rejection. Effect of conversion from cyclosporin to azathioprine therapy. Transpl Int. 1988;1(4):205-208.

48. Vincenti F, et al. Belatacept and long-term outcomes in kidney transplantation. N Engl J Med. 2016;374(4):333-343.

49. Baron D, et al. A common gene signature across multiple studies relate biomarkers and functional regulation in tolerance to renal allograft. Kidney Int. 2015;87(5):984-995.

50. Murphy SP, Porrett PM, Turka LA. Innate immunity in transplant tolerance and rejection. Immunol Rev. 2011;241(1):39-48.

51. Zhang PL, et al. Monocyte-mediated acute renal rejection after combined treatment with preoperative Campath-1H (alemtuzumab) and postoperative immunosuppression. Ann Clin Lab Sci. 2004;34(2):209-213.

52. Tinckam KJ, Djurdjev O, Magil AB. Glomerular monocytes predict worse outcomes after acute renal allograft rejection independent of C4d status. Kidney Int. 2005;68(4):1866-1874.

53. Bergler T, et al. Infiltration of macrophages correlates with severity of allograft rejection and outcome in human kidney transplantation. PLoS ONE. 2016;11(6):e0156900.

54. Colvin MM, et al. Antibody-mediated rejection in cardiac transplantation: emerging knowledge in diagnosis and management: a scientific statement from the American Heart Association. Circulation. 2015;131(18):1608-1639.

55. Hayde N, et al. Increased intragraft rejection-associated gene transcripts in patients with donor-specific antibodies and normal biopsies. Kidney Int. 2014;86(3):600-609.

56. Hidalgo LG, et al. NK cell transcripts and NK cells in kidney biopsies from patients with donor-specific antibodies: evidence for NK cell involvement in antibody-mediated rejection. Am J Transplant. 2010;10(8):1812-1822.

57. Chu Z, et al. Primed macrophages directly and specifically reject allografts. Cell Mol Immunol. 2020;17(3):237-246.

58. van den Bosch TP, Kannegieter NM, Hesselink DA, Baan CC, Rowshani AT. Targeting the monocyte-macrophage lineage in solid organ transplantation. Front Immunol. 2017;8:153.

59. Denes A, Lopez-Castejon G, Brough D. Caspase-1: is IL-1 just the tip of the ICEberg? Cell Death Dis. 2012;3:e338.

60. Todd JL, Palmer SM. Danger signals in regulating the immune response to solid organ transplantation. J Clin Invest. 2017;127(7):2464-2472.

61. Hong BJ, et al. Inflammasome activation involved in early inflammation reaction after liver transplantation. Immunol Lett. 2017;190:265-271.

62. Haubert D, et al. Vav1 GEF activity is required for T cell mediated allograft rejection. Transpl Immunol. 2012;26(4):212-219.

63. Zhang Q, Fairchild RL, Reich MB, Miller GG. Inhibition of Src kinases combined with CD40 ligand blockade prolongs murine cardiac allograft survival. Transplantation. 2005;80(8):1112-1120.

64. Wei C, et al. Genomic analysis of kidney allograft injury identifies hematopoietic cell kinase as a key driver of renal fibrosis. J Am Soc Nephrol. 2017;28(5):1385-1393.

65. Eskandary F, et al. A randomized trial of bortezomib in late antibody-mediated kidney transplant rejection. J Am Soc Nephrol. 2018;29(2):591-605

66. Budde K, et al. Sotrastaurin, a novel small molecule inhibiting protein kinase C: first clinical results in renal-transplant recipients. Am J Transplant. 2010;10(3):571-581. 
67. Parker GA, Picut CA. Immune functioning in non lymphoid organs: the liver. Toxicol Pathol. 2012;40(2):237-247.

68. Martin TR, Frevert CW. Innate immunity in the lungs. Proc Am Thorac Soc. 2005;2(5):403-411.

69. Metz LM, Eliasziw M. Trial of minocycline in clinically isolated syndrome of multiple sclerosis. N Engl J Med. 2017;377(8):789.

70. Chen M, et al. Minocycline inhibits caspase-1 and caspase-3 expression and delays mortality in a transgenic mouse model of Huntington disease. Nat Med. 2000;6(7):797-801.

71. Scarabelli TM, et al. Minocycline inhibits caspase activation and reactivation, increases the ratio of XIAP to smac/DIABLO, and reduces the mitochondrial leakage of cytochrome C and smac/DIABLO. J Am Coll Cardiol. 2004;43(5):865-874.

72. Pang T, Wang J, Benicky J, Saavedra JM. Minocycline ameliorates LPS-induced inflammation in human monocytes by novel mechanisms including LOX-1, Nur77 and LITAF inhibition. Biochim Biophys Acta. 2012;1820(4):503-510.

73. Dunston CR, Griffiths HR, Lambert PA, Staddon S, Vernallis AB. Proteomic analysis of the anti-inflammatory action of minocycline. Proteomics. 2011;11(1):42-51.

74. Kobayashi K, et al. Minocycline selectively inhibits M1 polarization of microglia. Cell Death Dis. 2013;4:e525.

75. Kloppenburg M, et al. The influence of tetracyclines on T cell activation. Clin Exp Immunol. 1995;102(3):635-641.

76. Giuliani F, Hader W, Yong VW. Minocycline attenuates T cell and microglia activity to impair cytokine production in T cell-microglia interaction. J Leukoc Biol. 2005;78(1):135-143.

77. Andes D, Craig WA. Animal model pharmacokinetics and pharmacodynamics: a critical review. Int J Antimicrob Agents. 2002;19(4):261-268.

78. Agwuh KN, MacGowan A. Pharmacokinetics and pharmacodynamics of the tetracyclines including glycylcyclines. $J$ Antimicrob Chemother. 2006;58(2):256-265.

79. Wohlfahrtova M, Tycova I, Honsova E, Lodererova A, Viklicky O. Molecular patterns of subclinical and clinical rejection of kidney allograft: quantity matters. Kidney Blood Press Res. 2015;40(3):244-257.

80. Kurian SM, et al. Orthogonal comparison of molecular signatures of kidney transplants with subclinical and clinical acute rejection: equivalent performance is agnostic to both technology and platform. Am J Transplant. 2017;17(8):2103-2116.

81. Glover FT. Handbook of Approximation Algorithms and Metaheuristics. Taylor \& Francis Group, LLC; 2007.

82. Korb KN, Nicholson AE. Bayesian Artificial Intelligence. Chapman Hall;2004.

83. Iorio F, et al. Discovery of drug mode of action and drug repositioning from transcriptional responses. Proc Natl Acad Sci U S A. 2010;107(33):14621-14626.

84. Niimi M. The technique for heterotopic cardiac transplantation in mice: experience of 3000 operations by one surgeon. $J$ Heart Lung Transplant. 2001;20(10):1123-1128.

85. Szot GL, et al. Different mechanisms of cardiac allograft rejection in wildtype and CD28-deficient mice. Am J Transplant. 2001;1(1):38-46.

86. Barker-Haliski ML, et al. Acute treatment with minocycline, but not valproic acid, improves long-term behavioral outcomes in the Theiler's virus model of temporal lobe epilepsy. Epilepsia. 2016;57(12):1958-1967.

87. Hahn JN, Kaushik DK, Mishra MK, Wang J, Silva C, Yong VW. Impact of minocycline on extracellular matrix metalloprotein ase inducer, a factor implicated in multiple sclerosis immunopathogenesis. J Immunol. 2016;197(10):3850-3860.

88. Xia D, Shen K, Zhong W, Pan H. Administration of minocycline ameliorates damage in a renal ischemia/reperfusion injury model. Clin Invest Med. 2011;34(2):E55-E63.

89. Lin H, et al. Long-term acceptance of major histocompatibility complex mismatched cardiac allografts induced by CTLA4Ig plus donor-specific transfusion. J Exp Med. 1993;178(5):1801-1806.

90. Uehara M, et al. Regulation of T cell alloimmunity by PI3K $\gamma$ and PI3K $\delta$. Nat Commun. 2017;8(1):951.

91. Superina RA, Peugh WN, Wood KJ, Morris PJ. Assessment of primarily vascularized cardiac allografts in mice. Transplantation. 1986;42(2):226-227. 